ISSN (print): 1698-6180. ISSN (online): 1886-7995

www.ucm.es/info/estratig/journal.htm

Journal of Iberian Geology 36 (2) 2010: 253-274

doi:10.5209/rev_JIGE.2010.v36.n2.11

\title{
Choristoderes and the freshwater assemblages of Laurasia
}

\author{
Coristoderos y las asociaciones de agua dulce de Laurasia
}

\author{
R. Matsumoto, S. E. Evans \\ Department of Cell and Developmental Biology, University College London, Gower Street, London, \\ WC1E 6BT, England \\ ucgarym@ucl.ac.uk; ucgasue@ucl.ac.uk
}

Received: 11/11/09 / Accepted: 30/06/10

\begin{abstract}
Choristoderes are freshwater aquatic diapsid reptiles of uncertain phylogenetic position. Although the lineage probably diverged from other diapsids in the Permo-Triassic, choristoderes apparently never reached Gondwana. Within Laurasia, however, they have been recovered from Japan, China, Mongolia, Trans-Baikal Russia, Central Asia, Western Europe, and North America, reaching extreme northern latitudes. Setting aside controversial Triassic records, their known temporal range currently extends from the Middle Jurassic (Britain, Kyrgyzstan) to the Miocene (Czech Republic). However, although small choristoderes are known to span the entirety of this period, the larger, more derived neochoristoderes are recorded only from the Early Cretaceous through to the earliest Eocene. The gavial-like neochoristodere Champsosaurus is the most familiar taxon, characterised by large size, a long rostrum and flared temporal fenestrae, but research over the last three decades has revealed many new genera and exposed an unexpected diversity in terms of body size (small to relatively large), neck length (long v. short) and skull morphology (longirostrine v. brevirostrine, open v. closed lower temporal fenestrae). Typically choristoderes occur as part of a mesic assemblage that includes fish, lizards, mammals, turtles, frogs, salamanders, small dinosaurs and, usually, crocodiles. At maturity, Jurassic choristoderes were generally smaller than co-occuring crocodiles and were relatively unspecialized postcranially. The Early Cretaceous of Asia saw a dramatic diversification of choristoderes, including the appearance of much larger neochoristoderes. Perhaps significantly, the relevant Asian horizons (e.g. Yixian Formation, China; Okurodani and Kuwajima formations, Japan) yield no crocodiles. In Late Cretaceous and Paleogene horizons however, across Euramerica, large gavial-like neochoristoderes came to share freshwater ecosystems with a diversity of crocodiles, the neochoristoderes apparently occupying a specialist (gavial-like) piscivorous niche as long as it was available and resources were adequate.
\end{abstract}

Keywords: Choristodera, Paleobiogeography, Fauna, Laurasia, Crocodylomorpha 
Resumen

Los Coristoderos son reptiles diápsidos acuáticos con posición filogenética incierta. A pesar de que el linaje probablemente divergió del resto de diápsidos en el Permo-Triásico, los coristoderos aparentemente nunca alcanzaron Gondwana. En Laurasia, sin embargo, se han registrado restos en Japón, China, Mongolia, la región Trans-Baikal de Rusia, Asia Central, Europa occidental y Norteamérica, alcanzando latitudes septentrionales extremas. Si evitamos el registro controvertido del Triásico, su rango temporal conocido se extiende desde el Jurásico Medio (Islas Británica, Kirguizistán), al Mioceno (Republica Checa). Sin embargo, a pesar de que los coristoderos pequeños se sabe que existieron a lo largo de todo este periodo, los más grandes, los neocoristoderos derivados se registran sólo desde el Cretácico temprano hasta el comienzo del Eoceno. Entre los neocoristoderos el de tipo gavial, Champsosaurus, es uno de los taxones más conocidos, caracterizado por su gran talla, un rostro alargado y amplias fosas temporales, sin embargo, las investigaciones desarrolladas durante las tres últimas décadas han revelado muchos géneros nuevos y puesto de manifiesto una diversidad inesperada en relación con su tamaño corporal (de pequeño a relativamente grande), la longitud del cuello( largo-corto) y la morfología del cráneo (longirostros-brevirostros; o con ventanas temporales inferiores cerradas). Habitualmente los coristoderos forman parte de asociaciones mésicas que incluyen a peces, lagartos, mamíferos, tortugas, salamandras, pequeños dinosaurios, y habitualmente cocodrilos. Al alcanzar la madurez, los coristoderos jurásicos fueron generalmente más pequeños que los cocodrilos de la misma asociación, y además con pocas especializaciones postcraneales. En el Cretácico temprano de Asia se dieron las condiciones para una extraordinaria diversificación de los coristoderos, incluyendo la aparición de formas de neocoristoderos de porte mucho mayor. De modo significativo, en los importantes depósitos asiáticos como los de la Formación Yixian, China; o los de las Formaciones Okurodani and Kuwajima, Japón, los cocodrilos están muy posiblemente ausentes. En horizontes del Cretácico Superior y del Paleógeno, sin embargo, y a lo largo de Suramérica, las formas de neocoristoderos de tipo gavial compartieron ecosistemas de agua dulce con una diversidad de cocodrilos, los neocoristoderos aparentemente ocuparían un nicho piscívoro especializado (de tipo gavial) en tanto que disponían de recursos adecuados.

Palabras clave: Choristodera, Paleobiogeografía, Fauna, Laurasia, Crocodylomorpha.

\section{Introduction}

Choristodera was erected by Cope (1876) to encompass Champsosaurus, a new fresh water reptile from the Upper Cretaceous Judith River beds of Montana, North America. A year later, Gervais (1877) described Simoedosaurus from the Upper Paleocene at Cernay, near Rheims, France. These two genera were the only representatives of Choristodera for more than a century, but over the last three decades, new discoveries have extended the temporal and geographical range of the group, and provided increased understanding of its diversity. Choristoderes have been found across Laurasia, from Japan in the east to Alberta in the west (e.g. Evans and Hecht 1993; Gao and Fox, 1998; Matsumoto et al., 2007, 2009) in deposits ranging in age from Middle Jurassic to Miocene. However, despite the improvement in the record, the position of choristoderes within Diapsida remains uncertain. Some analyses have suggested that choristoderes are basal Archosauromorpha (e.g. Evans, 1988; Gauthier et al., 1988), but others have placed them alternatively as the sister group of Archosauromorpha (e.g. DeBraga and Rieppel, 1997), of Archosauromorpha +Lepidosauromorpha (e.g. Evans, 1988; Gao and Fox, 1998; Dilkes, 1998), or of Euryapsida within an expanded Lepidosauromorpha (e.g. Müller, 2004). Resolution of their phylogenetic position requires a better understanding of the early history of the group, but each of the above hypotheses predicts that choristoderes had separated from their (undetermined) sister group by the end of the Permian.

Eleven choristoderan genera are currently considered valid. Of these, Champsosaurus and Simoedosaurus (Late Cretaceous-earliest Eocene, Euramerica), Ikechosaurus and Tchoiria (Cretaceous, China and Mongolia, Efimov, 1975; Sigogneau-Russell, 1981) are medium-sized (2-5 $\mathrm{m}$ total length, skulls up to $700 \mathrm{~mm}$ from rostral tip to occiput) (Fig. 1), gavialiform reptiles with cordiform skulls, long rostra, and short necks. All recent analyses support the placement of these four genera in a clade, Neochoristodera (Evans and Manabe, 1999; Gao and Fox, 1998; Matsumoto et al., 2007, 2009; Skutchas, 2008). The more basal 'non-neochoristoderes' are smaller (0.3-1.0m) and more diverse in their morphology. Cteniogenys (MiddleLate Jurassic, Euramerica, Evans 1990), Lazarussuchus (Oligocene-Miocene, Europe, Hecht, 1992; Evans and Klembara, 2005), Monjurosuchus (Early Cretaceous, China and Japan, Gao et al., 2000; Matsumoto et al., 2007), and Philydrosaurus (Early Cretaceous, China, Gao and Fox, 2005) are essentially lizard-like choristoderes with short necks and either open (Cteniogenys) or closed (the other three) lower temporal fenestrae, whereas the Early Cretaceous Shokawa (Japan, Evans and Manabe, 1999) and Hyphalosaurus (China, Gao et al., 1999), the Hyphalosauridae of Gao et al. (2008), are nothosauriform with elongated necks. Khurendukhosaurus (Early Cretaceous, Mongolia, Sigogneau-Russell and Efimov, 1984, Skutchas 2008; Matsumoto et al., 2009) is of similar/ or slightly larger size to Hyphalosaurus (holotype, IVPP V11075) 
(similar in dorsal centrum length but femur twice as long: Matsumoto pers. obs. 2008; Matsumoto et al., 2009), but is known only from fragmentary material and its skull and body shape (long or short necked) are not yet known. The Early Cretaceous Mongolian Irenosaurus (Efimov, 1979) is based on isolated postcranial bones that lack sufficient diagnostic characters to differentiate them from Tchoiria (Evans and Hecht, 1993; Efimov and Storrs, 2000) and the choristoderan status of the European Triassic Pachystropheus (Storrs and Gower, 1993; Storrs et al., 1996) is equivocal. Most recently, the genus Liaoxisaurus was named on the basis of a new specimen from the Jiufotang Formation of China (Gao et al., 2005), but it is closely similar to Ikechosaurus pijiagouensis from the same horizon and its validity as a distinct taxon is in doubt.
Our concept of Choristodera has thus changed substantially in recent years. As it currently stands, the fossil record of Choristodera suggests there was a gradual Jurassic/ Cretaceous increase in maximum size, coupled with an increase in morphological disparity, particularly with respect to the neck and skull. This resulted in several distinct morphotypes (e.g. small short-necked brevirostrine; small long-necked brevirostrine; large short-necked longirostrine), some of which paralleled those of contemporaneous crocodiles.

Choristoderes are typically found in association with a diverse freshwater assemblage, most members of which, including choristoderes, survived the end-Cretaceous extinction. However, their presence and their level of morphological diversity in different localities shows con-

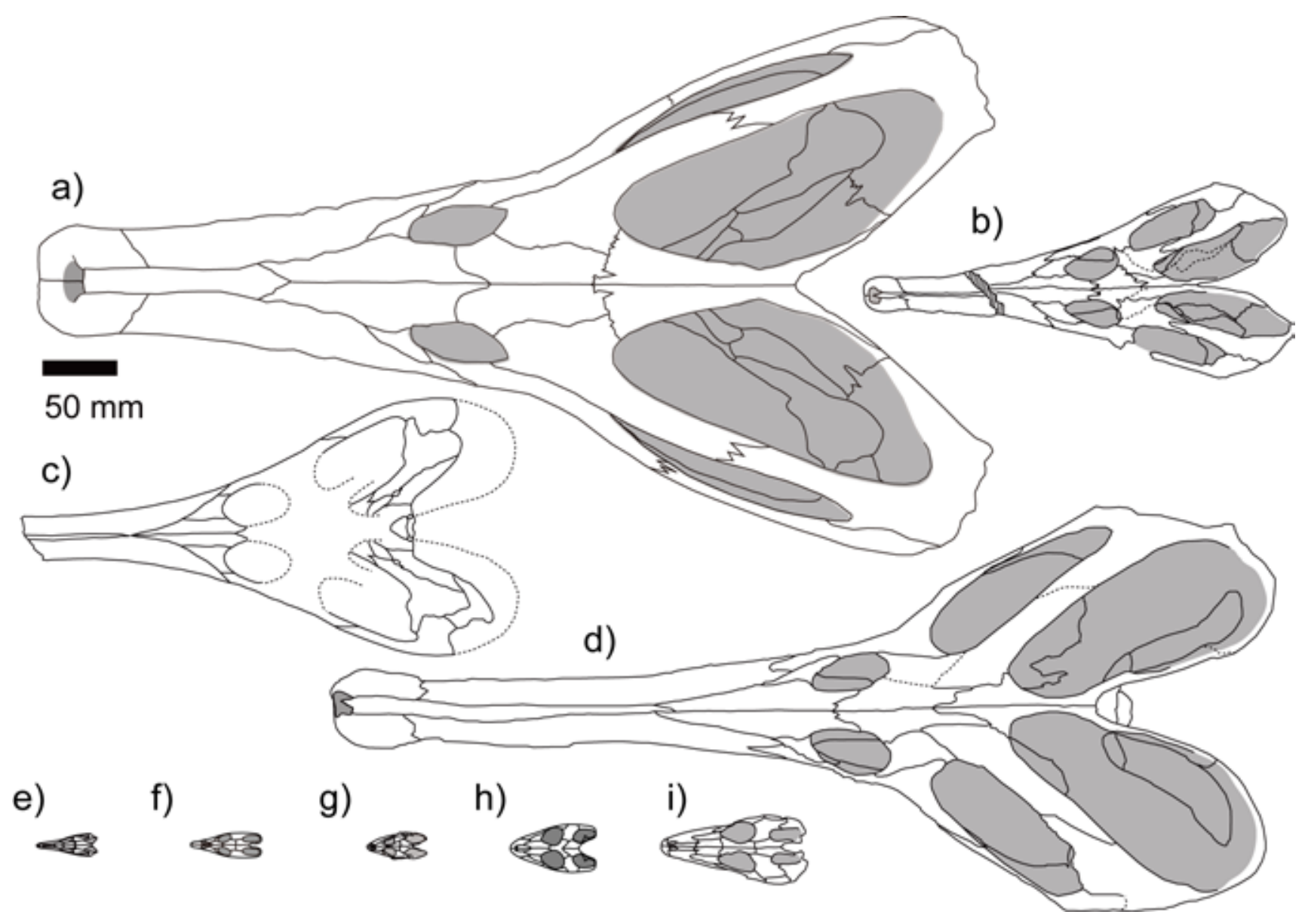

Fig. 1.- Choristoderan skulls drawn to scale: neochoristoderan -a) Simoedosaurus lemoinei (modified from Sigogneau-Russell and Russell, 1978) -b) Ikechosaurus pijiagouensis (IVPP V13283) -c) Tchoiria namsarai (redrawn from Efimov, 1975) -d) Champsosaurus gigas (SMM P77.33.24): non-neochoristoderan -e) Cteniogenys sp. (Evans, 1990)-f) Lazarussuchus inexpectatus (Hecht, 1992) -g) Hyphalosaurus baitaigouensis (redrawn from Gao and Ksepka, 2008) -h) Monjurosuchus splendens (Matsumoto et al., 2007) -i) Philydrosaurus proseilus (Gao and Fox, 2005).

Fig. 1.- Cráneos de Choristodera dibujados a escala: Neochoristodera -a) Simoedosaurus lemoinei (modificado de Sigogneau-Russell and Russell, 1978) -b) Ikechosaurus pijiagouensis (IVPP V13283) -c) Tchoiria namsarai (redibujado a partir de Efimov, 1975) -d) Champsosaurus gigas (SMM P77.33.24): non-Neochoristodera -e) Cteniogenys sp. (Evans, 1990)-f) Lazarussuchus inexpectatus (Hecht, 1992) - g) Hyphalosaurus baitaigouensis (redibujado a partir de Gao and Ksepka, 2008) - $\mathrm{h}$ ) Monjurosuchus splendens (Matsumoto et al., 2007) -i) Philydrosaurus proseilus (Gao and Fox, 2005). 
siderable variation, that may be indicative of underlying biological or ecological signals. Moreover, on current evidence, choristoderes became extinct in the Miocene while other members of the assemblage survived. Here we review choristoderan distribution and diversity from the Jurassic to the Miocene, and offer a preliminary analysis of the possible ecological relationship between choristoderes and other aquatic taxa, notably crocodiles with which they are most often compared.

\subsection{Institutional abbreviations}

GUI, Guimarota collections, Freie Universität, Berlin, Germany; IVPP V, Institute of Vertebrate Paleontology and Paleoanthropology, Beijing, China; SMNS Staatliches Museum für Naturkunde, Stuttgart, Germany; SMM, The Science Museum Minnesota, St. Paul, Minnesota, USA; UALVP, Laboratory for Vertebrate Paleontology, Department of Biological Science, University of Alberta, Edmonton, Canada.

\section{Temporal and geographical distribution}

\subsection{Triassic}

Although the Triassic genus Pachystropheus (England, Germany) has been referred to the Choristodera (e.g. Storrs and Gower, 1993; Storrs et al., 1996), it is too poorly known to be attributed with any confidence. The most diagnostic features of choristoderes are in their skulls, and there is no confirmed skull material for Pachystropheus.

\subsection{Jurassic}

The first definitive records of choristoderes are from the Middle Jurassic of Europe and Asia. Cteniogenys is a small, probably amphibious, choristodere from the Bathonian of England (e.g. Kirtlington Mammal Bed, Oxfordshire, England, Evans, 1990) and Scotland (Skye, Evans and Waldman, 1996). It is also recorded from the Kimmeridgian of Portugal (Guimarota, Seiffert, 1973; Evans, 1989) and North America (Brushy Basin Member, Morrison Formation, South Dakota, Wyoming and Utah: Gilmore, 1928; Evans, 1990; Chure and Evans, 1998; Foster and Trujillo, 2000). There is unnamed (but broadly similar) material from the Callovian Balanbansai Svita, Fergana Basin, of Kyrgyzstan (Averianov et al., 2006). Plotted on a paleoclimate map (Scotese, 2000), most of these records are in relatively warm temperate areas (Fig. 2-a). Although the Morrison Formation falls broad- ly into the arid climate zone, it covered a huge area and encompassed a range of palaeoenvironments, including wetlands suitable for small aquatic reptiles (Turner and Peterson, 2004). Interestingly, Cteniogenys is not evenly distributed through the Morrison (Brushy Basin Member) microvertebrate assemblages (Chure and Evans, 1998). It seems to be most common in the northern part of the formation (most notably at Quarry 9, Como Bluff), representing coastal swamp environments, but rare (Dinosaur National Monument) or absent (Fruita Paleontological area) on the Colorado Plateau (interior continental water bodies) (Chure and Evans, 1998). This may be significant because the British Bathonian localities and Guimarota both represent coastal freshwater-brackish lagoonal environments (e.g., Schudack, 2000), and the Balabansai Svita of the Fergana Depression has been described as transitional from terrestrial to marginal marine (Averianov et al., 2005).

\subsection{Lower Cretaceous}

Fossil deposits in the Lower Cretaceous of Asia (Eastern Russia, Inner Mongolia, China, Japan) have yielded the greatest known choristodere diversity, with at least seven recorded genera representing all known choristoderan morphotypes (Fig. 2-b): the long-necked Japanese Shokawa (Evans and Manabe, 1999) and Chinese Hyphalosaurus (Gao et al., 1999; Ji et al., 2004; Gao and Ksepka, 2008); the longirostrine gavial-like neochoristoderes Tchoiria (Mongolia, Efimov, 1979) and Ikechosaurus (Mongolia, China, Brinkman and Dong, 1993; Liu, 2004); the small lizard-like Monjurosuchus (China, Japan, Gao et al., 2000; Matsumoto et al., 2007) and Philydrosaurus (China, Gao and Fox, 2005; Gao et al., 2007); and the problematic Khurendukhosaurus (Mongolia, Sigogneau-Russell and Efimov, 1984; Skutschas, 2008; Matsumoto et al., 2009). At this time, Eastern Eurasia was divided into two paleophytogeographic provinces: a northern Siberian-Canadian Region (Vakhrameev, 1978), with Tetori-type floras at its southern margin (Kimura, 1979) and a temperate to humid climate; and a southern Euro-Sinian Region (Vakhrameev, 1978) with Ryosekitype floras (Kimura, 1979) and a suggested subtropical to tropical climate, with an annual dry season. All major choristodere localities occur in the northern province, in continental wetland (e.g. Kuwajima and Okurodani formations), fluvial (e.g. Khuren Dukh) or lacustrine (e.g. Yixian Formation) environments; they have not been reported from the southern province (e.g. the semi-arid fluvial ecosystem of the Khorat Group in Thailand [Meesook, 2000]), although several semi-aquatic fresh- 
water crocodiles (goniopholidids Sunosuchus, Goniopholis, and Siamosuchus, and the neosuchian Khoratosuchus) are known from the area (e.g. Lauprasert et al., 2009).

The Lower Cretaceous record for the rest of Laurasia is restricted to a partial femur, possibly neochoristoderan, from the Barremian Yellow Cat Member of the Cedar Mountain Formation of Utah (Britt et al., 2006). Palaeoenvironmental reconstructions for the Yellow Cat Formation (Kirkland et al., 1999) suggest a semiarid monsoonal environment, but one with enough standing water to support a stable freshwater community. The overlying Mussentuchit Formation contains a more diverse tetrapod assemblage, but there is no record of choristoderes.

\subsection{Upper Cretaceous}

In sharp contrast to the Lower Cretaceous, there is currently no record of choristoderes in the Upper Cretaceous of East Asia. This may reflect a lack of suitable depositional environments. Most Late Cretaceous fossil reptiles from China and Mongolia come from arid terrestrial environments. For choristoderes, the focus shifts to North America where there are abundant records of the gavial-like neochoristodere Champsosaurus in Utah, Wyoming, Montana, North Dakota, Alberta, Saskatchewan and the Canadian Arctic (Fig. 2-c). These localities, including those of the Arctic (Tarduno et al., 1998; Vandermark et al., 2007), were mostly located in lowlying river channels and deltaic deposits bordering the Western Interior Seaway; the climate was warm. This distribution may represent an ecological preference, or simply a prevalence of this type of environment within the Late Cretaceous rock record (e.g. Markwick, 1998). Putative records of non-neochoristoderes are limited to the Oldman and the Dinosaur Park formations, Alberta (Irvine locality and Dinosaur Provincial Park). These small specimens were assigned to Cteniogenys (Gao and Fox, 1998), but the material (incomplete dentaries and maxillae) is not sufficiently complete or diagnostic for generic identification (Matsumoto et al., 2009), especially with such a large age gap.

A single vertebra from the Lower Campanian of Austria (Gosau Beds, Muthmannsdorf; Buffetaut, 1989) demonstrates the presence of choristoderes in the Late Cretaceous of Europe. The fossil bearing horizon has been interpreted as a brackish estuarine environment (Sachs and Hornhung, 2006), with a humid, paratropical climate (Herman and Kvacek, 2007). Given the rarity of the choristodere material in the deposit, it may have been transported from further upstream.

\subsection{Paleocene to Miocene}

Champsosaurus survived the end-Cretaceous extinction and in the Paleocene was joined by another neochoristodere, Simoedosaurus. Conditions were temperate and neochoristoderes continued to be well represented in North America (Fig. 3-a), with limited European and Asian records. In Europe, Champsosaurus and Simoedosaurus have been recorded from littoral and infralittoral deposits at Erquelinnes, Belgium (Sigogneau-Russell and de Heinzelin, 1979) and from Mont Berru, France (Sigogneau-Russell and Russell, 1978; Sigogneau-Russell, 1985), respectively, and there is undescribed material of Champsosaurus from the latter locality (Matsumoto pers. obs. 2008). Postcranial elements of Simoedosaurus have also been reported from Upper Paleocene-Early Eocene coastal deposits (Dzhylga 1a-1b) in southern Kazakhstan (Averianov, 2005), where conditions were warmer and more humid.

In North America, the faunal transition from the Paleocene to the Eocene (Clarkforkian-Watsachian) has been well studied in continuous sections such as those of the Bighorn Basin, Wyoming (Bartels, 1983; Gunnel et al., 1993). Choristodere material is abundant in the Tiffanian and early Clarkforkian horizons in the Fort Union Formation of Wyoming, but is quite rare in the younger (early Eocene, Watsachian) Willwood Formation. Bartels (1983) attributed this decline to local effects (a reduction in the number and size of the streams), but this was also an interval of major faunal change associated with elevated temperatures. These are also the last records of neochoristoderes, and may mark the extinction of the clade (Gingerich, 2000; Gunnell et al., 1993; Bartels, 1983). However, non-neochoristoderes, having effectively disappeared from the record in the Early Cretaceous, unexpectedly reappeared in the Paleogene of Europe (France, Germany, Czech Republic) in the form of the small, lizard-like Lazarussuchus (Hecht, 1992; Evans and Klembara, 2005; Böhme, 2008; Evans et al., unpublished). These small reptiles were in continental lake environments. Their last record in Europe is from the Lower Miocene of the Czech Republic, in a warm (subtropical) wet environment (Fig. 3-b).

In summary, Choristodera were distributed throughout Laurasia from the Jurassic to the Miocene in warm temperate-paratropical localities. However, the group is notably absent or poorly represented in some localities and time periods, for example the Lower Cretaceous of Europe and the Upper Cretaceous of Asia. As so often with 


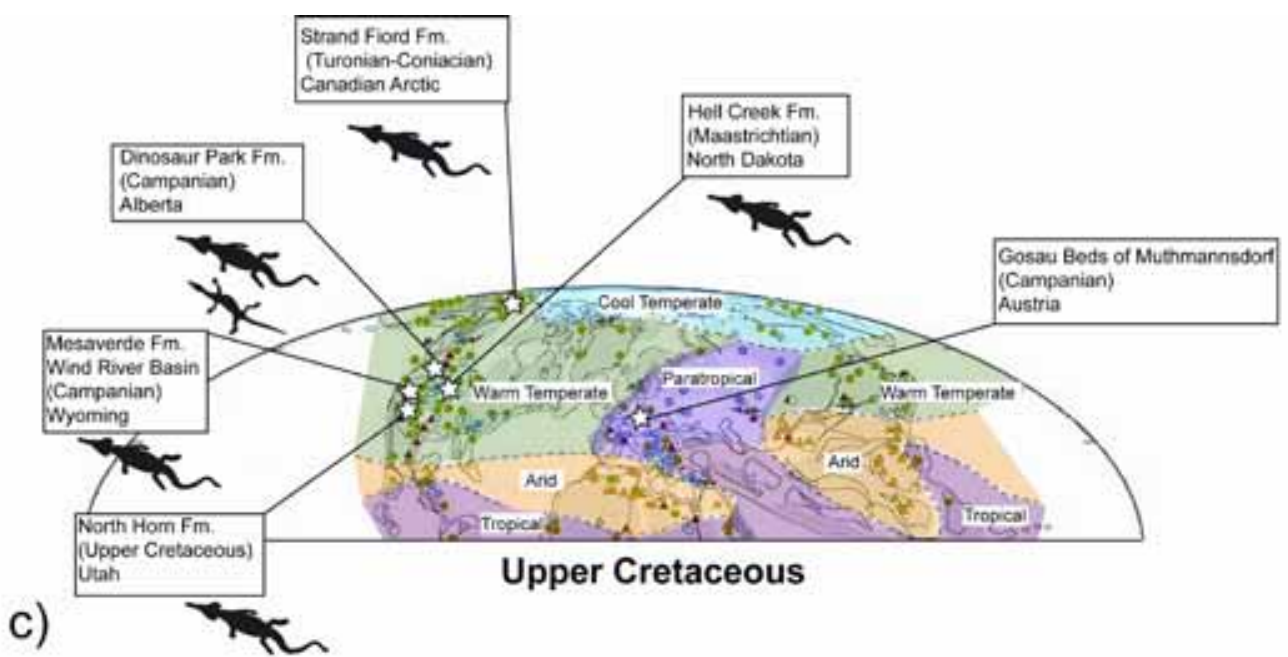

c)

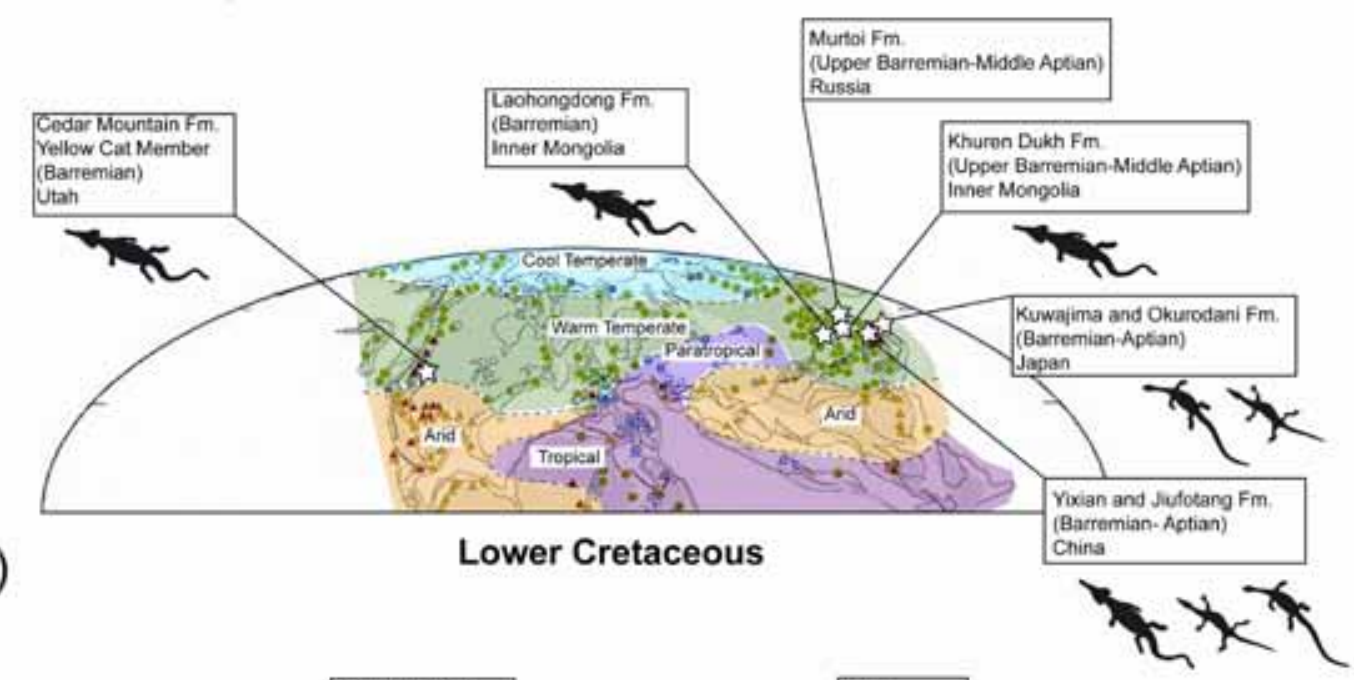

b)

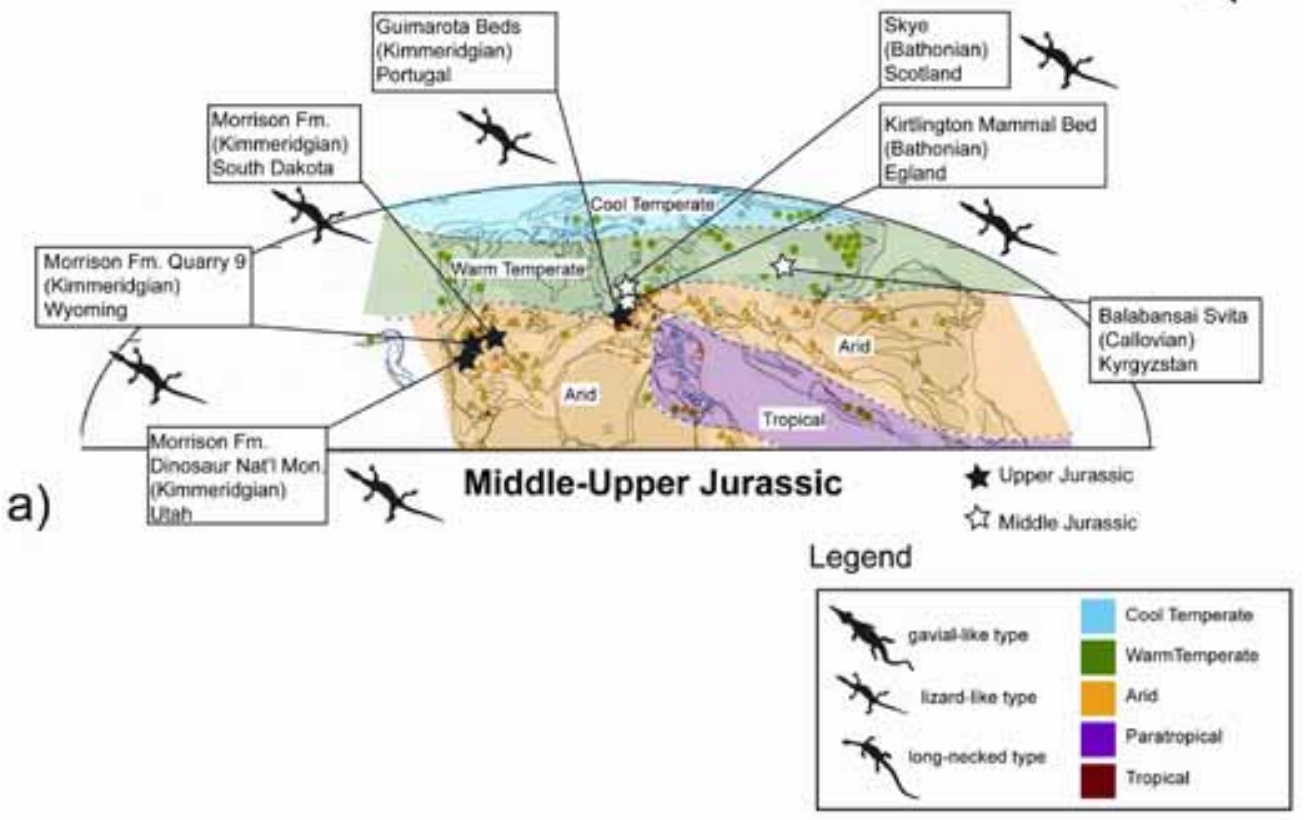

Fig. 2.- Paleogeographical distribution of Choristodera during the Mesozoic: plotted on climate history maps (modified from Scotese, 2002). -a) Middle-Upper Jurassic. -b) Lower Cretaceous. -c) Upper Cretaceous.

Fig. 2.- Distribución paleobiogeográfica de Choristodera durante el Mesozoico en relación con los mapas de la historia climática modificado de Scotese (2002). -a) Jurásico Medio-Superior. -b) Cretácico Inferior. -c) Cretácico Superior. 


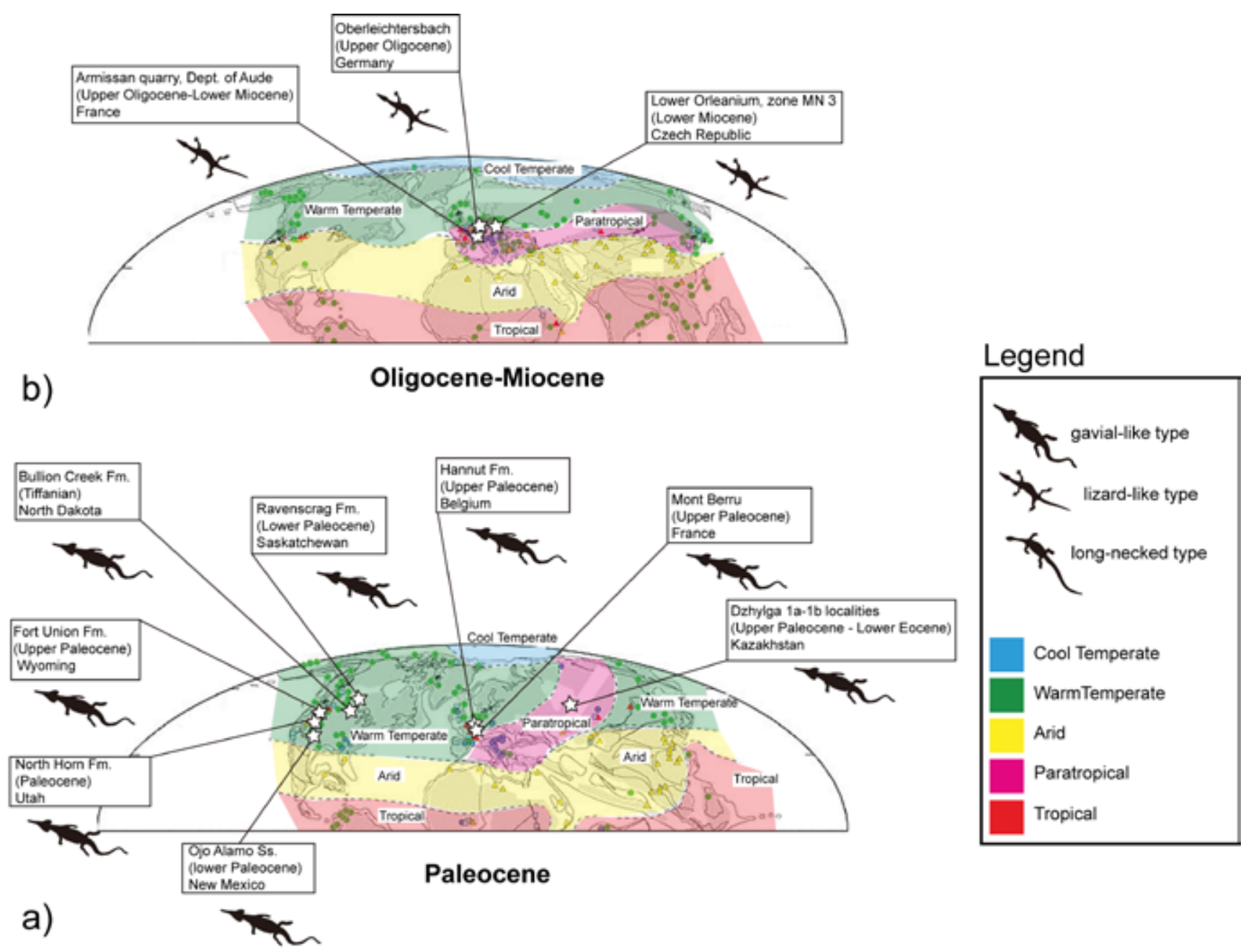

Fig. 3.- Paleogeographical distribution of Choristodera during the Cenozoic: plotted on climate history maps (modified from Scotese, 2002). -a) Paleocene. -b) Oligocene-Miocene.

Fig. 3.- Distribución paleogeográfica de Choristodera durnate el Cenozoico en relación con los mapas históricos climáticos modificados de Scotese (2002). -a) Paleoceno. -b) Oligoceno-Mioceno.

the fossil record, the challenge is to determine whether these absences are simply an artifact of sampling, preservation and taphonomy, or are real absences that require explanation and may provide an insight into the biology, lifestyle, or ecological interactions of the group.

\section{Faunal associations and ecological relationships with crocodiles}

\subsection{Assemblage composition}

A comparison of well-known Mesozoic to Cenozoic assemblages (Fig. 4) demonstrates that choristoderes occur in mesic depositional environments that are rich in vertebrates, especially fresh water and amphibious animals such as fish, frogs, salamanders, turtles and crocodiles (Fig. 4-a). Typically, these assemblages contain a greater diversity of crocodiles than choristoderes (Fig 5; Table 2-4) although they did not necessarily all live together. For example, the North Horn Formation (Upper CretaceousLower Paleocene, Utah, USA) has reportedly yielded five crocodile taxa (possibly an overestimate as the count is based on isolated teeth) and one choristodere. However, representatives of the two groups are found in different localities (Cifelli et al., 1999a) and this may reflect differences in habitat preference/tolerance or, possibly, some form of competitive exclusion. Similarly, six crocodiles have been recorded from the Morrison Formation (Fig 5; Table 2), with one small choristodere (Cteniogenys), but three of the crocodiles (the 'Fruita crocodile', Hallopus, and Hoplosuchus) are basal forms that were mainly or fully terrestrial and come from localities on the Colorado Plateau where Cteniogenys is rare or absent. In addition, several very productive horizons/localities in the 
a)

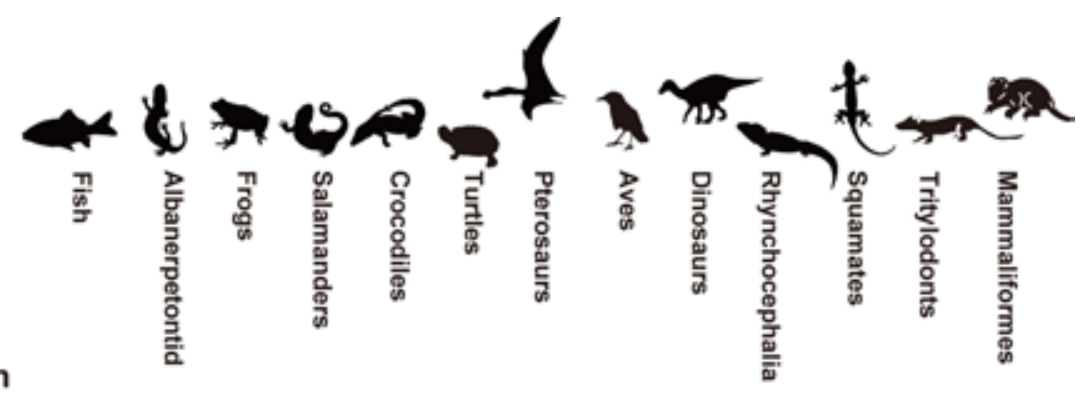

\begin{tabular}{|c|c|c|c|c|c|c|c|c|c|c|c|c|c|c|c|c|c|}
\hline \multirow{4}{*}{$\begin{array}{l}\Phi \\
\stackrel{5}{\Phi} \\
\stackrel{O}{0} \\
\frac{\Phi}{\pi} \\
0\end{array}$} & \multirow{4}{*}{$\begin{array}{l}\stackrel{Ð}{\mathrm{D}} \\
\stackrel{\mathrm{D}}{\mathrm{O}} \\
\sum_{1}\end{array}$} & EU & $\begin{array}{l}\text { Czech } \\
\text { Republic }\end{array}$ & $\begin{array}{l}\text { Lower Orleanium } \\
\text { (Lower Miocene) }\end{array}$ & 0 & - & O & 0 & O & O & \multicolumn{3}{|c|}{ O } & - & \multicolumn{2}{|l|}{ O } & \multirow{2}{*}{$\begin{array}{l}0 \\
-\end{array}$} \\
\hline & & EU & Germany & $\begin{array}{l}\text { Oberleichtersbach } \\
\text { (Oligocene) }\end{array}$ & 0 & - & O & 0 & 0 & 0 & & - & & - & 0 & & \\
\hline & & NA & Wyoming & $\begin{array}{l}\text { Fort Union Fm. } \\
\text { (Upper Paleocene) }\end{array}$ & 0 & - & $\mathrm{O}$ & 0 & 0 & 0 & & 0 & & - & 0 & & O \\
\hline & & NA & North Dakota & $\begin{array}{l}\text { Bullion Creek Fm. } \\
\text { (Tiffanian) }\end{array}$ & O & - & O & O & O & $\mathrm{O}$ & & O & & - & O & & O \\
\hline \multirow{5}{*}{ 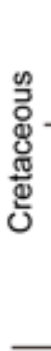 } & \multirow{5}{*}{$\begin{array}{l}\frac{.}{\Phi} \\
\text { 응 } \\
\\
\end{array}$} & NA & Utah & $\begin{array}{l}\text { North Horn Fm. } \\
\text { (Maastrichtian) }\end{array}$ & 0 & - & O & - & 0 & O & - & - & O & - & O & - & 0 \\
\hline & & NA & Alberta & $\begin{array}{l}\text { Oldman Fm. } \\
\text { (Campanian) }\end{array}$ & 0 & 0 & $\mathrm{O}$ & O & O & 0 & - & 0 & $\mathrm{O}$ & - & 0 & - & - \\
\hline & & AS & Japan & $\begin{array}{l}\text { Kuwajima Fm. } \\
\text { (Barremian-Aptian) }\end{array}$ & 0 & - & 0 & $?$ & - & O & 0 & 0 & O & - & 0 & O & $\mathrm{O}$ \\
\hline & & AS & China & $\begin{array}{l}\text { Yixian Fm. } \\
\text { (Barremian-Aptian) }\end{array}$ & 0 & - & $\mathrm{O}$ & 0 & - & 0 & 0 & 0 & 0 & - & 0 & - & 0 \\
\hline & & NA & Utah & $\begin{array}{l}\text { Cedar Mountain Fm. } \\
\text { Yellow Cat Member } \\
\text { (Barremian) }\end{array}$ & 0 & - & - & - & O & 0 & 0 & - & 0 & 0 & - & - & - \\
\hline \multirow{4}{*}{ 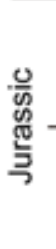 } & \multirow{2}{*}{$\begin{array}{l}\text { 음 } \\
\text { 응 }\end{array}$} & NA & Wyoming & $\begin{array}{l}\text { Morrison Fm. } \\
\text { (Kimmeridgian) }\end{array}$ & O & - & 0 & O & O & O & O & - & 0 & 0 & O & - & O \\
\hline & & EU & Portugal & $\begin{array}{l}\text { Guimarota Beds } \\
\text { (Kimmeridgian) }\end{array}$ & 0 & 0 & 0 & 0 & O & 0 & 0 & 0 & 0 & 0 & 0 & - & 0 \\
\hline & \multirow{2}{*}{$\begin{array}{l}\frac{\Phi}{D} \\
\frac{D}{\Sigma} \\
\Sigma\end{array}$} & AS & Kyrgyzstan & $\begin{array}{l}\text { Balabansai Svita } \\
\text { (Callovian) }\end{array}$ & 0 & - & - & 0 & 0 & 0 & 0 & - & O & - & 0 & - & 0 \\
\hline & & EU & England & $\begin{array}{l}\text { Kirtlington Mammal Bed } \\
\text { (Bathonian) }\end{array}$ & O & $\mathrm{O}$ & 0 & O & 0 & 0 & O & - & 0 & 0 & 0 & O & 0 \\
\hline
\end{tabular}

b)

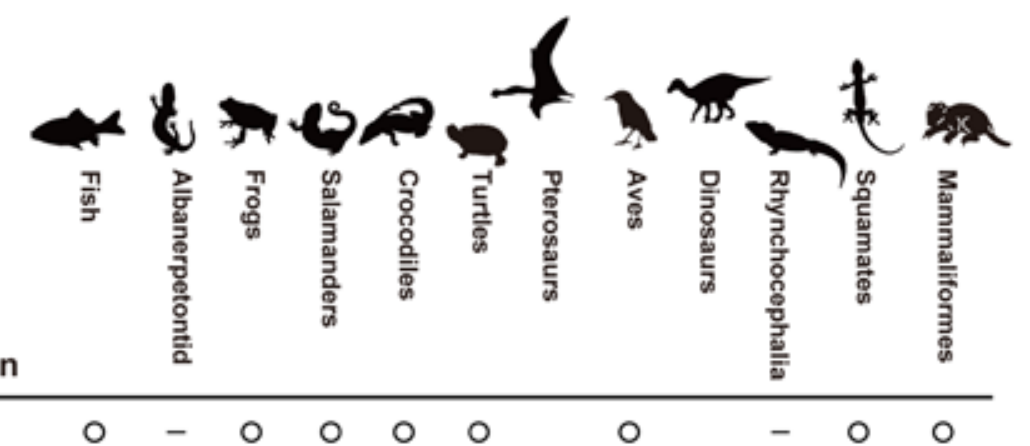

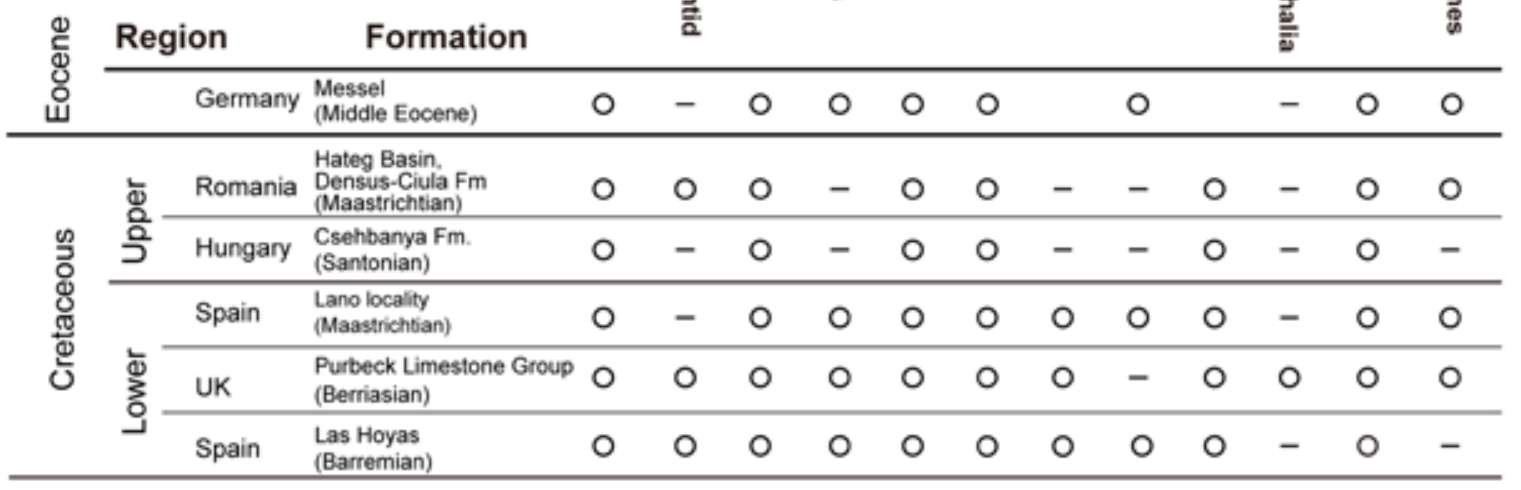

Fig. 4.- Assemblage composition through the Mesozoic and Cenozoic. -a) well studied choristoderan localities -b) European microvertebrate localities where choristoderes are not known.

Fig. 4.- Composición de las asociaciones a lo largo del Mesozoico y Cenozoico. -a) localidades bien estudiadas con coristoderos. -b) localidades Europeas con microvertebrados donde no se han registrado coristoderos. 
Cretaceous and Eocene of Europe yield crocodiles but no choristoderes (Fig. 4-b) despite an otherwise generally similar associated freshwater fauna. These include: the Purbeck Limestone Group (Berriasian) of England (three valid crocodile genera, e.g. Salisbury, 2002); the limestones of the La Huergina Formation (Barremian) at Las Hoyas, Spain (at least five crocodile genera, Ortega et al., 1999, Buscalioni and Fregenal-Martínez, 2006); and the Wessex Formation (Barremian) of England (eight crocodile genera, Sweetman, 2009, but based mainly on teeth); as well as the Middle Eocene beds of Messel, Germany (seven crocodile genera, Morlo et al., 2004).

By contrast, in those Lower Cretaceous Asian horizons that contain the greatest diversity of choristoderes (e.g. Yixian Formation, China; Kuwajima and Okurodani formations, Japan; Fig. 5; Table 4), crocodiles are conspicuously absent. Whether this is an ecological or temporal phenomenon is not clear, as possible goniopholid crocodile remains have been reported (Azuma, 2003), from the somewhat drier (Yabe et al., 2003) late Hauterivian to

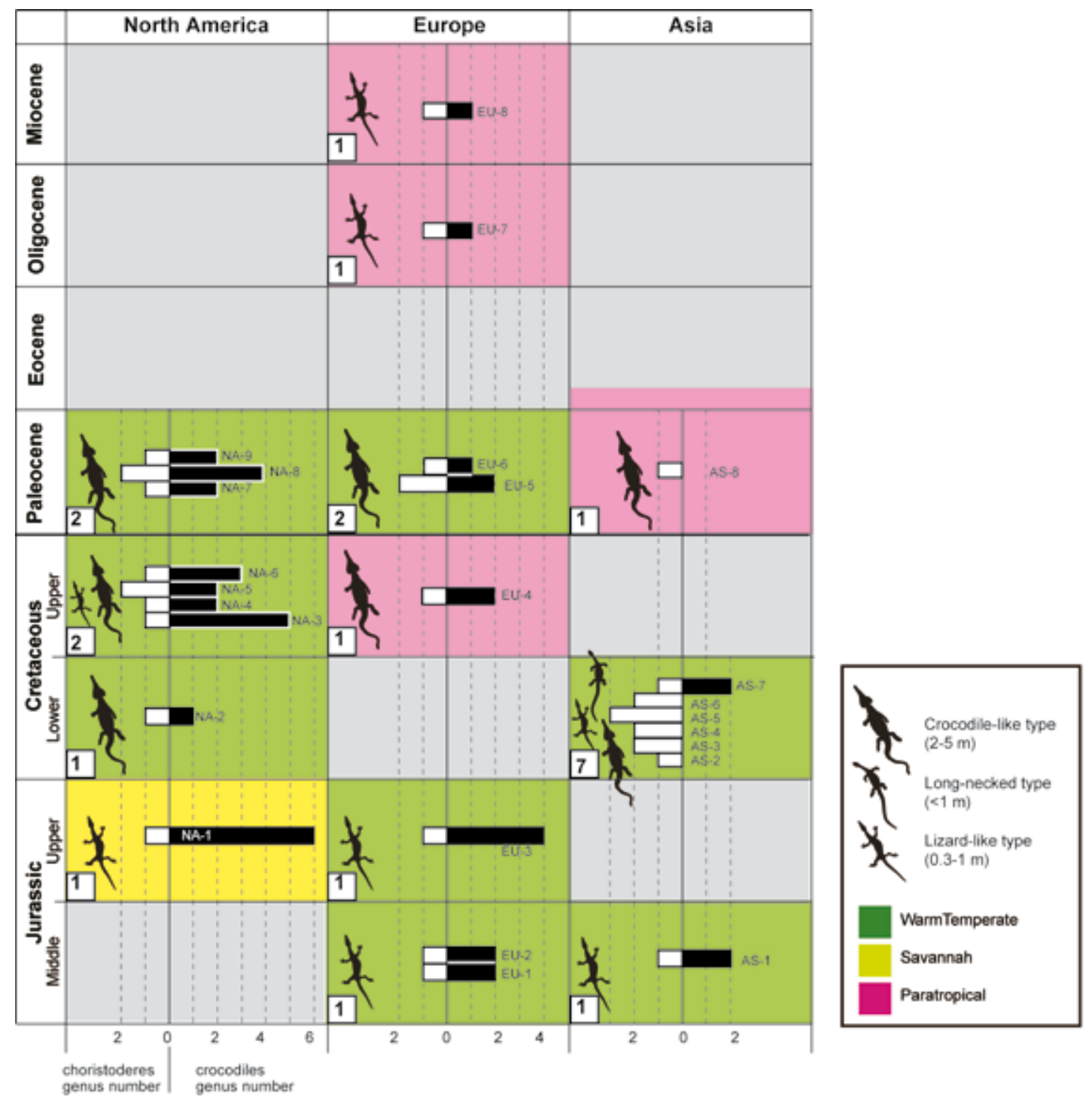

Fig. 5.- Comparison of relative numbers of choristoderes and crocodiles (genera). Each bar indicates one horizon: choristoderes are in white and crocodiles in black. The horizon number corresponds to that in table 2-4: North America (NA) 1-9 in Table 2; Europe (EU) 1-9 in Table 3; Asia (AS) 1-8 in Table 4. The number in the left hand corner of each box indicates the total number of choristodere genera for that region and period.

Fig. 5.- Comparación entre el número relativo de coristoderos y de cocodrilos (géneros). Cada barra indica un horizonte: coristoderos se representan en blanco y cocodrilos en negro. El número del horizonte corresponde a los que se expresan en la tabla 2-4: Norte America (NA) 1-9 en la Tabla 2; Europa (EU) 1-9 en la Tabla 3; Asia (AS) 1-8 en la Tabla 4. El número en el extremo izquierdo de cada caja indica el número total de géneros de coristoderos para esa región y periodo. 


\begin{tabular}{|c|c|c|c|c|c|c|c|c|c|c|c|}
\hline & & Region & Choristoderes & Skull & Size & References & Region & Crocodiles & Skull & Size & References \\
\hline \multicolumn{2}{|l|}{$\begin{array}{l}\text { Miocene-Upper } \\
\text { Oligocnene }\end{array}$} & EU & Lazarussuchus & $0.05 \mathrm{~m}$ & $0.3 \mathrm{~m}$ & Hecht, 1992 & EU & Diplocynodon & & $0.3-1.5 \mathrm{~m}$ & $\begin{array}{l}\text { Piras and Buscalioni, } \\
2006\end{array}$ \\
\hline \multirow[t]{6}{*}{ Paleocene } & & NA & Champsosaurus gigas & $0.49 \mathrm{~m}$ & $3.5 \mathrm{~m}$ & Erickson, 1985 & NA & Leidyosuchus & & $4 \mathrm{~m}$ & Erickson, 1982 \\
\hline & & EU & C. dolloi & $0.65 \mathrm{~m}$ & $4 \mathrm{~m}$ & $\begin{array}{l}\text { Sigogneau-Russell } \\
\text { and Heinzelin, } 1979\end{array}$ & NA & Wannaganosuchus & & $1 \mathrm{~m}$ & Erickson, 1982 \\
\hline & & NA & Simoedosaurus dakotensis & $0.71 \mathrm{~m}$ & $5 \mathrm{~m}$ & Erickson, 1987 & NA & Ceratosuchus & $0.23 \mathrm{~m}$ & $2 \mathrm{~m}$ & Bartels, 1984 \\
\hline & & EU & Simoedosaurus sp. & $0.52 \mathrm{~m}$ & $4 \mathrm{~m}$ & SMNS 59026 & NA & Allognathosuchus & $0.24 \mathrm{~m}$ & $2 \mathrm{~m}$ & Simpson, 1930 \\
\hline & & & & & & & EU & Eosuchus & & $3.5 \mathrm{~m}$ & Delfino et al., 2005 \\
\hline & & & & & & & EU & Asiatosuchus sp. & & $>4 \mathrm{~m}$ & Russell et al., 1990 \\
\hline \multirow[t]{11}{*}{ Cretaceous } & Upper & NA & Champsosaurus natator & $0.5 \mathrm{~m}$ & $3.6 \mathrm{~m}$ & Russell, 1956 & $\overline{N A}$ & Brachychampsa montana & $0.39 \mathrm{~m}$ & $3 \mathrm{~m}$ & Norell et al., 1994 \\
\hline & & & C. laramiensis & $0.41 \mathrm{~m}$ & $3.0 \mathrm{~m}$ & Brown, 1905 & NA & Leidyosuchus sternbergi & $0.34 \mathrm{~m}$ & $3 \mathrm{~m}$ & Brochu, 1997 \\
\hline & & & C. ambulator & $0.4 \mathrm{~m}$ & $3 \mathrm{~m}$ & Brown, 1905 & NA & Thoracosaurus neocesariensis & & $2.5 \mathrm{~m}$ & $\begin{array}{l}\text { Brandalise de Andrade, } \\
\text { personal communication, } \\
\text { August } 2009\end{array}$ \\
\hline & & & & & & & EU & Doratodon carcharidens & & $?$ & \\
\hline & Lower & AS & Ikechosaurus pijiagoensis & $0.27 \mathrm{~m}$ & $1.7 \mathrm{~m}$ & Liu, 2004 & AS & Theriosuchus & & $0.5 \mathrm{~m}$ & $\begin{array}{l}\text { Schwarz and Salisbury, } \\
2005\end{array}$ \\
\hline & & AS & Tchoiria namsarai & $0.3 \mathrm{~m}$ & $2 \mathrm{~m}$ & undescribed material & AS & Shantungosuchus & & $0.3 \mathrm{~m}$ & Wu et al., 1994 \\
\hline & & AS & Khurendkuhosaurus & $?$ & $1 \mathrm{~m} ?$ & $\begin{array}{l}\text { Matsumoto et al., } \\
2009\end{array}$ & & & & & \\
\hline & & AS & Philydrosaurus proseilus & $0.1 \mathrm{~m}$ & $<1 \mathrm{~m} ?$ & Gao et al., 2007 & & & & & \\
\hline & & AS & Monjurosuchus splendens & $0.06 \mathrm{~m}$ & $\sim 0.5 \mathrm{~m}$ & $\begin{array}{l}\text { Gao and Li, 2006; } \\
\text { Gao et al., } 2000\end{array}$ & & & & & \\
\hline & & AS & Hyphalosaurus lingyuanensis & $0.06 \mathrm{~m}$ & $1 \mathrm{~m}$ & $\begin{array}{l}\text { Holotype (IVPP } \\
\text { V11705) }\end{array}$ & & & & & \\
\hline & & AS & Shokawa ikoi & $?$ & $>0.6 \mathrm{~m}$ & $\begin{array}{l}\text { Evans and Manabe, } \\
1999\end{array}$ & & & & & \\
\hline \multirow[t]{10}{*}{ Jurassic } & Middle- & EU & Cteniogenys sp. & $0.04 \mathrm{~m}$ & $\sim 0.3 \mathrm{~m}$ & Evans, 1990 & $E U$ & Macelognathus & & $3 \mathrm{~m}$ & Hups et al., 2005 \\
\hline & Upper & /NA & & & & & EU & Machimosaurus & & $9.5 \mathrm{~m}$ & Charig, 1976 \\
\hline & & & & & & & EU/NA & Goniopholis & & $3 \mathrm{~m}$ & $\begin{array}{l}\text { Schwarz and Salisbury, } \\
2005\end{array}$ \\
\hline & & & & & & & EU & Lusitanisuchus & & $0.4 \mathrm{~m}$ & $\begin{array}{l}\text { Schwarz and Fechner, } \\
2008\end{array}$ \\
\hline & & & & & & & EU & Theriosuchus & & $0.5 \mathrm{~m}$ & $\begin{array}{l}\text { Schwarz and Salisbury, } \\
2005\end{array}$ \\
\hline & & & & & & & EU & Eutretauranosuchus & & $3 \mathrm{~m}$ & Hups et al., 2005 \\
\hline & & & & & & & NA & Hoplosuchus kayi & & $0.2 \mathrm{~m}$ & \\
\hline & & & & & & & NA & Hallopus victor & & $0.6 \mathrm{~m}$ & Walker, 1970 \\
\hline & & & & & & & NA & "Fruitachamysa" & & $1 \mathrm{~m}$ & \\
\hline & & & & & & & AS & Sunosuchus sp. & $0.42 \mathrm{~m}$ & $3 \mathrm{~m}$ & Schellhorm et al., 2009 \\
\hline
\end{tabular}

Table 1.- Estimated total length (skull to tail tip) for choristoderes and crocodiles

Tabla 1.- Longitud total estimada (cráneo a cola) para coristoderos y cocodrilos.

late Aptian Kitadani Formation (e.g. Kusuhashi, 2008; Tsubamoto, 2004), which overlies the choristodere bearing Kuwajima and Okurodani formations in Japan (e.g. Fujita, 2003; Kusuhashi, 2008). To date, only one Asian horizon (the Barremian Laohongdong Formation, Inner Mongolia) is known to contain both choristoderes (Tchoiria) and crocodiles. Sha et al. (2006) report that crocodiles were also found in the Khuren Dukh Formation (Mongolia), but no further data is available and Averianov and Skutchas (2000) do not include crocodiles in their faunal list for this formation. Significantly, Buscalioni et al. (2003) have reported that the Cretaceous crocodile fauna of Asia was dominated by basal groups (protosuchids, gobisuchids, ziphosuchians) that were largely terrestrial in their habits.

\subsection{Comparison of size and rostral morphology in croco- diles and choristoderes}

Based on Table 1, Tables 2-4 present comparisons of body size for crocodiles and choristoderes in selected horizons where they co-occur; Fig. 6 compares rostral morphologies in the same horizons. Body size estimates (total length: tip of snout to tail tip) are taken mainly from the literature, as listed. However, where body size data is lacking, size has been estimated for crocodiles based on skull/ body length ratios for extant Crocodylus (Wermuth, 1964; Bellairs, 1969), and for choristoderes based on the proportions of a complete articulated skeleton of the neochoristodere Ikechosaurus pijiagouensis (IVPP V13283, Liu, 2004) with a head:body ratio of 1:6.2 (Table 4). As skull morphology and size change ontogenetically in crocodiles (Dodson, 1975), the skulls used in these estimates were restricted to mature specimens.

\section{Jurassic}

Early choristoderes were small. In most Jurassic horizons where choristoderes and crocodiles are found together (e.g. Middle Jurassic of UK and Kyrgyzstan; Upper Jurassic of Portugal, North America), the adult crocodiles (mainly goniopholids but also atoposaurids) are up to ten times larger $(\sim 2.5-3.0 \mathrm{~m})$ than the choristoderes (Cteniogenys: $\sim 0.3-0.5 \mathrm{~m})$. Recently discovered choristodere vertebral centra from the Balanbasai Svita, Fergana (Mid- 


\begin{tabular}{|c|c|c|c|c|c|c|c|c|c|c|}
\hline \multicolumn{2}{|l|}{ North America } & \multicolumn{2}{|c|}{ Horizon No. Formations } & \multirow[t]{2}{*}{$\begin{array}{l}\text { Skull } \\
\text { No. }\end{array}$} & \multirow{2}{*}{$\begin{array}{l}\text { Choristoderes } \\
\text { Champsosaurus gigas }\end{array}$} & \multirow{2}{*}{$\begin{array}{l}\text { Size } \\
3.5 \mathrm{~m}\end{array}$} & \multirow[t]{2}{*}{$\begin{array}{l}\text { Skull } \\
\text { No. }\end{array}$} & \multirow{2}{*}{$\begin{array}{l}\text { Crocodiles } \\
\text { Leidyosuchus formidabilis }\end{array}$} & \multirow{2}{*}{$\begin{array}{l}\text { Size } \\
3-4 \mathrm{~m}\end{array}$} & \multirow{2}{*}{$\begin{array}{l}\text { References } \\
\text { Erickson, } 1999\end{array}$} \\
\hline Paleocene & & NA-9 & North Dakota & & & & & & & \\
\hline & & & $\begin{array}{l}\text { Bullion Creek Fm. } \\
\text { (Tiffanian) }\end{array}$ & & Champsosaurus sp. & $3-4 \mathrm{~m}$ & & $\begin{array}{l}\text { Wannaganosuchus brachymanus } \\
\text { possibly juvenile of Allognathosuchus (Lucas and Estep, } \\
\text { 2000) }\end{array}$ & $1 \mathrm{~m}$ & \\
\hline & & $\overline{\mathrm{NA}-8}$ & Wyoming & ch-10 & Champsosaurus gigas & $3.5 \mathrm{~m}$ & cr-22 & Allognathosuchus (e.g. A. heterodon) & $2 \mathrm{~m}$ & Bartels, 1983 \\
\hline & & & Fort Union Fm. & ch-9 & Simoedosaurus sp. & $4-5 \mathrm{~m}$ & $\mathrm{cr}-21$ & Leidyosuchus formidabilis & $4 \mathrm{~m}$ & \\
\hline & & & (Upper Paleocene) & & & & $\mathrm{cr}-20$ & Ceratosuchus burdoshi & $2 \mathrm{~m}$ & \\
\hline & & & & & & & cr-19 & (Diplocynodon sp.) & $0.3-1.5 \mathrm{~m}$ & \\
\hline & & NA-7 & Utah & & Champsosaurus & $3-4 \mathrm{~m}$ & & Allognathosuchus & $2 \mathrm{~m}$ & Cifelli et al.,1999a \\
\hline & & & $\begin{array}{l}\text { North Horn Fm. } \\
\text { (Paleocene) }\end{array}$ & & & & & Leidyosuchus & $4 \mathrm{~m}$ & \\
\hline \multirow[t]{16}{*}{ Cretaceous } & Upper & NA-6 & North Dakota & ch-7 & Champsosaurus sp. & $3-4 \mathrm{~m}$ & cr-17 & Brachychampsa montana & $3 \mathrm{~m}$ & Pearson et al., 2002 \\
\hline & & & Hell Creek Fm. & & & & cr-16 & Leidyosuchus sternbergi (=Borealosuchus; Brochu, 1997) & $3-4 \mathrm{~m}$ & \\
\hline & & & (Maastrichtian) & & & & $\mathrm{cr}-15$ & Thoracosaurus neocesariensis & $2.5 \mathrm{~m}$ & \\
\hline & & $\overline{\mathrm{NA}-5}$ & Alberta & & Champsosaurus natator & $3-4 \mathrm{~m}$ & & Leidyosuchus canadensis & $3 \mathrm{~m}$ & Brinkman, 1990 \\
\hline & & & $\begin{array}{l}\text { Dinosaur Park Fm } \\
\text { (Campanian) }\end{array}$ & & non-Neochoristodera & $?$ & & Albertochampsa langstoni & & $\begin{array}{l}\text { Gao and Brinkman, } 2005 \\
\text { Wu, } 2005\end{array}$ \\
\hline & & NA-4 & Wyoming & & Champsosaurus sp. & $3-4 \mathrm{~m}$ & & Leidyosuchus sp. & $3-4 \mathrm{~m}$ & $\begin{array}{l}\text { DeMar and Breithaupt, } \\
2008\end{array}$ \\
\hline & & & $\begin{array}{l}\text { Mesaverde Fm. Wind River } \\
\text { Basin } \\
\text { (Campanian) }\end{array}$ & & & & & Alligatorinae & ? & \\
\hline & & NA-3 & Utah & & Champsosaurus sp. & $3-4 \mathrm{~m}$ & & ? Atoposauridae & $?$ & Cifelli et al.,1999a \\
\hline & & & North Horn Fm. & & & & & gen. and sp. Indet. & & \\
\hline & & & (Upper Cretaceous) & & & & & gen. and sp. Indet. & ? & \\
\hline & & & & & & & & ? Pholidosauridae & ? & \\
\hline & & & & & & & & Leidyosuchus sp. & $3-4 \mathrm{~m}$ & \\
\hline & & & & & & & & Allognathosuchus & $2 \mathrm{~m}$ & \\
\hline & Lower & NA-2 & Utah & ch-5 & Neochoristodera indet. & $?$ & cr-12 & Crocodylia indet. & $?$ & Britt et al., 2009 \\
\hline & & & $\begin{array}{l}\text { Cedar Mountain Fm. Yellow } \\
\text { Cat Member }\end{array}$ & & & & & & & Britt et al., 2006 \\
\hline & & & (Barremian) & & & & & & & Kirkland el al., 1999 \\
\hline \multirow[t]{6}{*}{$\begin{array}{l}\text { Jurassic } \\
\end{array}$} & Upper & NA-1 & Wyoming & ch-3 & Cteniogenys antiquus & $0.3 \mathrm{~m}$ & cr-8 & Goniopholis sp. & $3 \mathrm{~m}$ & Clark, 1994 \\
\hline & & & Morrison Fm. & & & & $\mathrm{cr}-7$ & Hoplosuchus kayi & $0.2 \mathrm{~m}$ & Chure, et al., 1998 \\
\hline & & & & & & & $c r-6$ & Hallopus victor & $0.6 \mathrm{~m}$ & Göhlich, et al., 2005 \\
\hline & & & (Kimmeridgian) & & & & cr-5 & Macelognathus vagans & $3 \mathrm{~m}$ & Hups, et al., 2005 \\
\hline & & & & & & & & Eutretauranosuchus & $2 \mathrm{~m}$ & \\
\hline & & & & & & & & "Fruitachampsa" & $1 \mathrm{~m}$ & \\
\hline
\end{tabular}

Table 2.- List of choristoderan localities/horizons with crocodiles in North America

Tabla 2.- Lista de localidades/horizontes de coristoderos con cocodrilos en Norte América.

dle Jurassic), Kyrgyzstan (Averianov et al., 2006), are of a similar size and morphology to those of Cteniogenys from the Morrison Formation, North America (Foster and Trujillo, 2000). Although, the body proportions of this Asian choristodere are still unknown, the total length would have been less than $1 \mathrm{~m}$ even if it was long-necked (by comparison with the largest specimens of the Lower Cretaceous Hyphalosaurus IVPP V11075). Two crocodiles are known from the Balanbasai Svita (Averianov et al., 2006). The first, the neosuchian Sunosuchus, has a total length of about $2.5 \mathrm{~m}$. The other is an undetermined thalattosuchian of unknown length. At Guimarota, Portugal, the Kimmeridgian lignites have yielded several crocodiles (Martin and Krebs, 2000). The largest is the longirostrine Machimosaurus, about 30 times the size of Cteniogenys, but this is an open sea crocodile (Krebs, 1967). Three crocodiles are of similar length to Cteniogenys: the poorly known Lusitanisuchus (0.4 m) (Schwarz and Fechner, 2008), Lisboasaurus estesi and the atoposaurid Theriosuchus (Schwarz and Salisbury, 2005: 0.5 $\mathrm{m})$. These three are relatively brevirostrine crocodiles (rostral length/ basal skull length $\sim 50 \%$ ), not dissimilar in overall skull shape to Cteniogenys (Evans, 1990), but they are primarily terrestrial. The Morrison Formation is similar, with both large aquatic crocodiles (up to $3 \mathrm{~m}$ : Eutretauranosuchus, Goniopholis spp., Macelognathus) and smaller terrestrial ones (0.2-1.0 m: Hallopus victor, Hoplosuchus kayi, and the 'Fruita crocodile') (Clark, 1994; Chure et al., 1998; Göhlich et al., 2005; Hups et al., 2005).

\section{Lower Cretaceous}

Crocodile teeth and osteoderms are recorded from the Yellow Cat Member of the Cedar Mountain Formation, in conjunction with the choristodere femur recorded above (Britt et al., 2006). A more diverse crocodile assemblage is known from the overlying Mussentuchit Formation (Cifelli et al., 1999b), but without choristoderes. The Barremian Laohongdong Formation, Inner Mongolia, is currently the only Lower Cretaceous deposit in Asia yielding both choristoderes (the large gavial-like Ikechosaurus sunailinae) and crocodiles (the protosuchian Shantungosuchus, $0.3 \mathrm{~m}$ : Wu et al., 1994; and the atoposaurid cf. Theriosuchus sp., $0.5 \mathrm{~m}$ : Wu et al., 1996, known also from 


\begin{tabular}{|c|c|c|c|c|c|c|c|c|c|c|}
\hline Europe & & $\begin{array}{l}\text { Horizon } \\
\text { No. }\end{array}$ & Formations & $\begin{array}{l}\text { Skull } \\
\text { No. }\end{array}$ & Choristoderes & Size & $\begin{array}{l}\text { Skull } \\
\text { No. }\end{array}$ & Crocodiles & Size & References \\
\hline Oligocene- & & EU-9 & Czech Republic & ch-14 & Lazarussuchus davoraki & $0.3 \mathrm{~m}$ & $\mathrm{cr}-25$ & Diplocynodon sp. & $0.3-1.5 \mathrm{~m}$ & Evans and Klembara, 2005 \\
\hline \multirow[t]{8}{*}{ Miocene } & & & $\begin{array}{l}\text { Lower Orleanium, zone MN } 3 \\
\text { Formation }\end{array}$ & & & & & & & \\
\hline & & & (Lower Miocene) & & & & & & & \\
\hline & & & France & & Lazarussuchus inexpectatus & $0.3 \mathrm{~m}$ & & $?$ & & Hecht, 1992 \\
\hline & & & Armissan quarry & & & & & & & \\
\hline & & & (Upper Oligocene-Lower Miocne) & & & & & & & \\
\hline & & EU-7 & Germany & ch-13 & Lazarussuchus sp. & & cr-24 & Diplocynodon sp. & $0.3-1.5 \mathrm{~m}$ & Böhme, 2008 \\
\hline & & & Oberleichtersbach & & & & & & & \\
\hline & & & (Upper Oligocene) & & & & & & & \\
\hline \multirow[t]{5}{*}{ Paleocene } & & EU-6 & Belgium & ch-11 & Champsosaurus dolloi & $4 \mathrm{~m}$ & cr-23 & Eosuchus leichei & $3.5 \mathrm{~m}$ & Delfino et al., 2005 \\
\hline & & & $\begin{array}{l}\text { Hannut Fm. } \\
\text { (Upper Paleocene) }\end{array}$ & & & & & & & \\
\hline & & EU-5 & France & & Simoedosaurus lemoinei & $4 \mathrm{~m}$ & & Asiatosuchus sp. & $>4 \mathrm{~m}$ & Sigogneau-Russell, 1985 \\
\hline & & & Mont Berru & & Champsosaurus & $3-4 m$ & & Borealosuchus sp. & & $\begin{array}{l}\text { Prasad and F de Lapparent } \\
\text { de Borin, } 2002\end{array}$ \\
\hline & & & & & & & & & & Martin, 2008 \\
\hline \multirow[t]{3}{*}{ Cretaceous } & Upper & EU-4 & Lower Austria & ch-8 & Choristodera indet. & $?$ & & Doratodon carcharidens & $?$ & Sachs and Hornung, 2006 \\
\hline & & & Gosau Group & & & & cr-18 & Alligatoridae indet. & $?$ & Buffetaut, 1979 \\
\hline & & & Lower Campanina & & & & & & & \\
\hline \multirow[t]{9}{*}{ Jurassic } & Upper & EU-3 & Portugal & ch-4 & Cteniogenys sp. & $0.3 \mathrm{~m}$ & $\mathrm{cr}-11$ & Machimosaurus & $9.5 \mathrm{~m}$ & Evans and Chure, 1998 \\
\hline & & & Guimarota Beds & & & & cr-10 & Goniopholis & $3 \mathrm{~m}$ & Martin and Krebs, 2000 \\
\hline & & & (Kimmeridgian) & & & & & Lusitanisuchus & $0.4 \mathrm{~m}$ & \\
\hline & & & & & & & $\mathrm{cr}-9$ & Theriosuchus & $0.5 \mathrm{~m}$ & \\
\hline & Middle & $\mathrm{EU}-2$ & England & ch-1 & Cteniogenys sp. & $0.3 \mathrm{~m}$ & $\mathrm{cr}-2$ & $\begin{array}{l}\text { Goniopholididae (cf. } \\
\text { Nannosuchus=?juvenile Goniopholis) }\end{array}$ & $?$ & Evans and Milner, 1994 \\
\hline & & & $\begin{array}{l}\text { Kirtlington Mammal bed } \\
\text { (Upper Bathonian) }\end{array}$ & & & & $\mathrm{cr}-1$ & Atoposauridae & $?$ & \\
\hline & & EU-1 & Scotland & & Cteniogenys sp. & $0.3 \mathrm{~m}$ & & Atoposauridae & $?$ & \\
\hline & & & Skye & & & & & Goniopholididae & $?$ & Evans and Waldman, 1996 \\
\hline & & & (Upper Bathonian) & & & & & & & \\
\hline
\end{tabular}

Table 3.- List of choristoderan localities/horizons with crocodiles in Europe

Tabla 3.- Lista de localidades/horizontes de coristoderos con cocodrilos en Europa.

Guimarota [see above]). Isolated larger (mature) specimens of I. sunailinae (e.g. IVPP V 1596.5) from the Laolonghuoze locality (Brinkman and Dong, 1993) are similar in size (centrum length $18.4 \mathrm{~mm}$ at cervical [C]3) to the adult of Ikechosaurus pijiagouensis (Liu, 2004) from the Jiufotang Formation of China (centrum length 17-18 $\mathrm{mm}$ through C2-C9; $1.7 \mathrm{~m}$ total body length). Thus, in the one Asian locality where crocodiles and choristoderes are known to co-occur, the choristoderes appear to have been larger. Moreover, as protosuchids and atoposaurids are considered to be primarily terrestrial (Brinkman, 1989; Schwarz and Salisbury, 2005; Wu et al., 1994), the two groups had different niches.

\section{Upper Cretaceous}

The isolated choristoderan vertebra from the Gosau Group of Muthmannsdorf (Lower Austria) is about 15 $\mathrm{mm}$ in length. This equates to a body length of about 2 $\mathrm{m}$, assuming neochoristoderan proportions (e.g. Ikechosaurus pijiagouensis: presacral centrum length $17-20 \mathrm{~mm}$, total length $\sim 2.0 \mathrm{~m}$ ). The Gosau bone bed has also yielded two crocodiles, the ziphosuchian Doratodon (Company et al., 2005) and Alligatoridae indet. (Buffetaut, 1979). At Muthmannsdorf, Doratodon is known only from cranial elements but there have been recent discoveries of the same genus from other localities and formations (Grigorescu et al., 1999; Company et al., 2005). It is a small, probably terrestrial ziphosuchian, possibly similar to the genus Bergisuchus in having a tall short-snouted skull (Company et al., 2005).

In North America, Champsosaurus is common at many localities and is typically found with more aquatic crowngroup crocodilians, most usually the similar-sized Leidyosuchus (some species of which have been placed in the genus Borealosuchus, Brochu, 1997). The two differ, however, in rostral morphology and thus, presumably, feeding habit. Champsosaurus has a long slender rostrum whereas that of Leidyosuchus-Borealosuchus is wider and has been described as 'generalised' (Brochu, 2001). In the Hell Creek Formation (Maastrichtian) of southwestern North Dakota and northwestern South Dakota, crocodiles with a wider range of rostral shapes are found with Champsosaurus (Pearson et al., 2002). The closest match is the longirostrine gavialoid Thoracosaurus (e.g. 


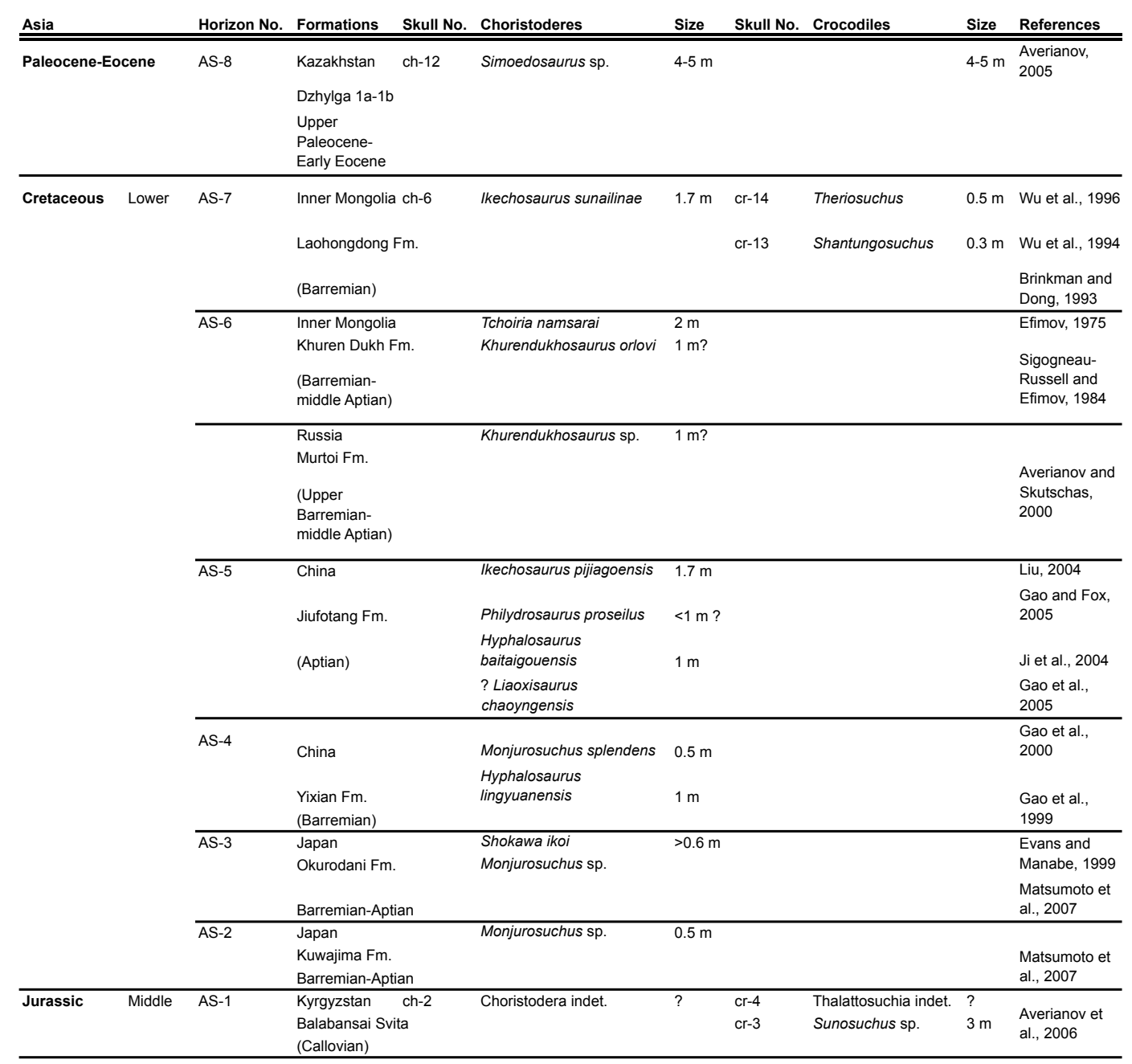

Table 4.- List of choristoderan localities/horizons with crocodiles in Asia

Tabla 4.- Lista de localidades/horizontes de coristoderos con cocodrilos en Asia.

Leidy, 1852), which is slightly smaller than Champsosaurus (Champsosaurus 3-4 m: Thoracosaurus $2.5 \mathrm{~m}$ ). However, although the remains of Thoracosaurus occur in the same layer as Champsosaurus, its bones are much rarer than those of other crocodiles (Pearson et al., 2002), suggesting they may be allochthonous. In support of this, Buscalioni et al. (2003) suggested Thoracosaurus might be a coastal dweller like other Gavialoidea (including Eosuchus, see below), although juveniles may have lived in freshwater environments as in the extant saltwater crocodile, Crocodylus porosus (Jouve et al., 2008).

Small incomplete choristoderan jaw elements have been reported from the Oldman and the Dinosaur Park formations of Alberta, Canada, in association with Champsosaurus and Leidyosuchus (Brinkman, 1990; Gao and Brinkman, 2005; Gao and Fox, 1998) The affinities of these specimens (e.g. UALVP29794; UALVP29795) are uncertain, but the reptiles from which they came, if adult, would have been of similar size to Portuguese (Guimarota) specimens of Cteniogenys (e.g. GUI. A.33, Seiffert, 1973).

\section{Paleocene-Eocene}

At some Paleocene localities, Champsosaurus ( 4 m) and Simoedosaurus ( $\sim \mathrm{m})$ occur together (e.g. MontBerru, France, Sigogneau-Russell, 1985, Matsumoto pers. obs. 2008; Fort Union Formation, Wyoming, USA, Bartels, 1983; Ravenscrag Formation, Saskatchewan, Canada, Gao and Fox, 1998). In the Fort Union Formation (Wyoming, Bartels, 1983), these neochoristoderes are recorded with three genera of crocodiles. Of these, the basal eusuchian Leidyosuchus-Borealosuchus is similar in size to the choristoderes, but the blunt-snouted alligatorines Allognathosuchus and Ceratosuchus are smaller. In the Ravenscrag Formation (Russell, 1974) and at Mont Berru (Martin, 2008), Leidyosuchus-Borealosuchus again occurs with the neochoristoderes, in conjunction with the large ( $\sim \mathrm{m}$ ) crocodyloid Asiatosuchus at Mont Berru (Prasad and Lapparent de Broin, 2002). In each deposit, the crocodiles and choristoderes differ in rostral morphology. By contrast, in the Hannut Formation, 


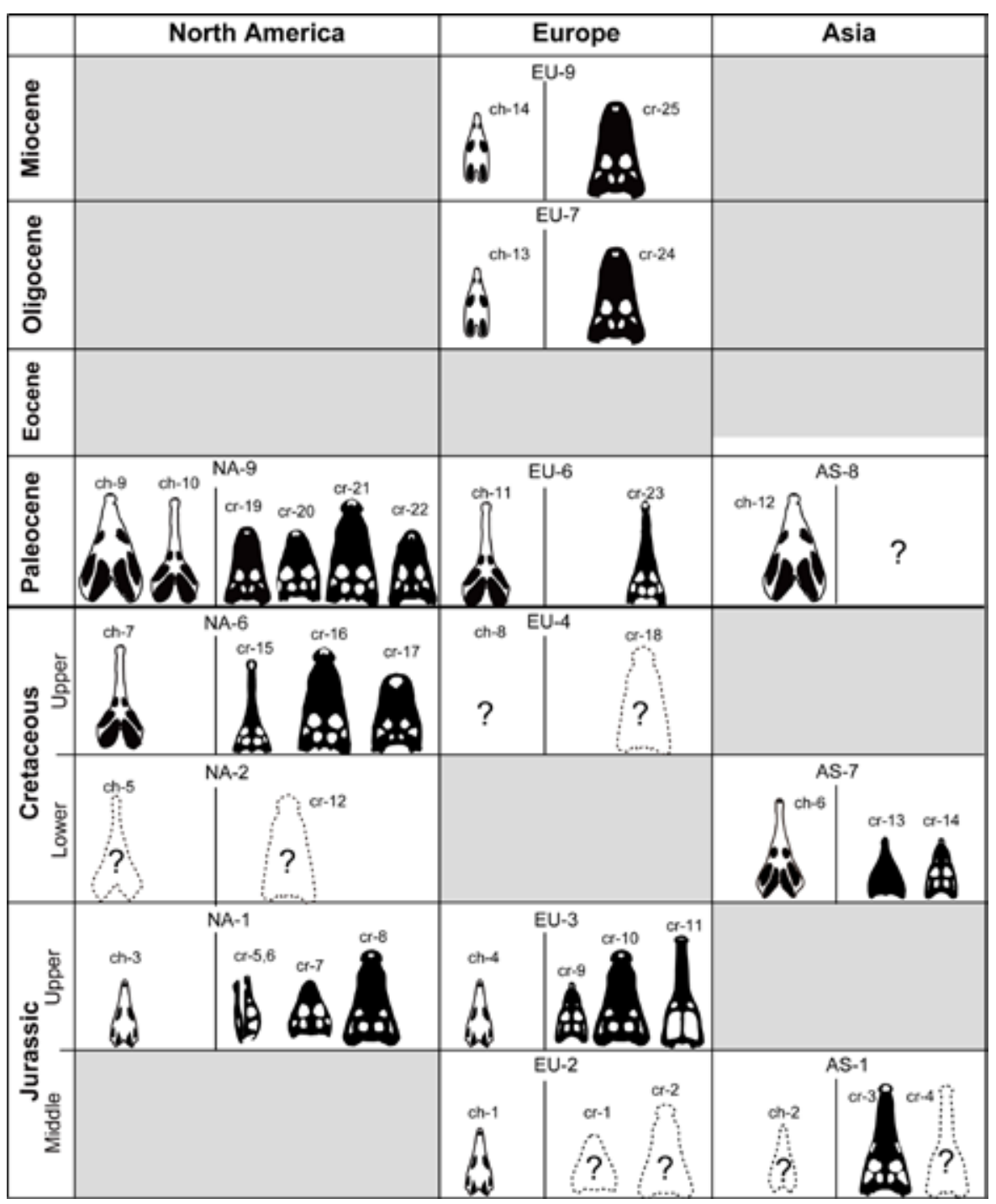

Fig. 6.- Skull morphology of choristoderes and crocodiles in selected horizons, white for choristoderes and black for crocodiles. The horizon numbers (e.g. NA-1) and skull numbers (e.g. ch-1 for choristoderes; cr-1 for crocodiles) correspond to those in Tables 2-4.

Fig. 6.- Morfología del cráneo de los coristoderos y de los cocodrilos en los horizontes seleccionados, en blanco para coristoderos y negro para cocodrilos. Los números en los horizontes (p.ej., NA-1) así como los números en los cráneos (p.ej., ch-1 para coristoderos; cr-1 para cocodrilos) corresponden a los indicados en las Tablas 2-4.

Belgium, Champsosaurus co-occurs with the gavialoid crocodile Eosuchus, but although they were both recovered from the same shallow marine layer (SigogneauRussell and de Heinzelin, 1979), they probably did not live in the same environment. The bones of Champsosaurus are rounded, suggesting they were transported into the deposit from upriver, whereas Eosuchus may be salt water tolerant (Delfino et al., 2005), as evidenced by the occurrence of the North American species of Eosuchus (Marsh, 1870) in marine deposits.

The Paleocene-Eocene Dzhylga 1a and 1b locality, Kazakhstan (Averianov, 2005), is the only Asian locality of this age to yield choristoderes. Crocodiles have not been recorded but the significance of this is limited, as the faunal assemblage as a whole is depauperate and lacks other freshwater components such as amphibians (Averianov, 2005).

\section{Oligocene-Miocene}

The only recorded post-Eocene choristodere is the small European (France, Czech Republic, Germany) Lazarussuchus (0.3 m body length, Hecht, 1992). At the Oligocene-Miocene French locality of Armissan, the fauna is poorly known and the presence of crocodiles is uncertain (Hecht, 1992). However, at Oberleichtersbach, Germany (Late Oligocene, Böhme, 2008) and Merkur, Czech 
Republic (Lower Miocene, Evans and Klembara 2005), Lazarussuchus is found with the much larger $(\sim \times 7)$ alligatoroid crocodile Diplocynodon (Fejfar and Schelich, 1994).

\section{Discussion}

\subsection{Choristoderes within freshwater assemblages}

Choristoderes were distributed across Laurasia in the Mesozoic and early Paleogene, typically in relatively warm, temperate climates (occasionally sub-tropical areas, Fig. 5), and in association with a diverse mesic vertebrate assemblage including fish, frogs, salamanders, turtles, and crocodiles (Fig. 4-a). Typically, crocodiles are more diverse than choristoderes (up to 6:1, Fig. 5), although allowance must be made for the fact that many basal crocodiles were predominantly terrestrial. However, there are some well-sampled localities in which either crocodiles (e.g. Lower Cretaceous of China, Japan) or choristoderes (e.g. Lower Cretaceous Purbeck Limestone Group and Wessex Formation, UK) are completely absent. This suggests either differences in habitat preference or some level of competition (whereby choristodere diversity was limited by the presence of aquatic crocodiles). Generally, the associated freshwater assemblage is similar whether the assemblage contains crocodiles, choristoderes or both, but there does seem to be a tendency for choristoderes to be found in low lying freshwater environments close to coastal margins, at least in the Jurassic, Late Cretaceous and Paleocene (although this may partially reflect the prevalence of this type of deposit). Climate and other physical conditions are likely to have played an important role in choristoderan distribution and possibly extinction (as they do for crocodiles, Markwick, 1998), and this needs to be analyzed more thoroughly than we have space to do here.

Basal, non-neochoristoderan taxa were small and were unlikely to have been in serious competition with the adults of contemporaneous crocodiles, most of which were either larger or occupied a different, more terrestrial, niche. The choristoderes are more likely to have competed for resources with a range of other freshwater animals including predatory fish, turtles, juvenile crocodiles and aquatic lizards, as well as other small choristoderes. Nonetheless, a recent study in central Africa (Luiselli et al., 1999) examined the ecological relationship between the small extant crocodile Osteolaemus and a similarsized potential competitor, the predatory lizard Varanus. The two reptiles could co-exist as long as prey was abundant, and this is likely to have been the case for the choristoderes in relation to other small aquatic predators.
With the evolution of the neochoristoderes, there may have been a greater potential for competition with crocodiles, particularly as neosuchian crocodiles were themselves also radiating into more aquatic niches. Neochoristoderes are generally equal, or nearly equal, in size to contemporaneous crocodiles, the only exception being in the Lower Cretaceous Laohongdong Formation of Inner Mongolia, where the neochoristodere Ikechosaurus sunailinae is nearly twice the size of the associated crocodiles. However, this is a special case as contemporaneous East Asian crocodiles mainly belong to basal groups (protosuchians, gobisuchidans and ziphosuchians, Buscalioni et al., 2003) that were primarily terrestrial.

Where several crocodiles, extinct or extant, co-occur they tend to differ morphologically, most notably in rostral shape. Rostral shape in crocodiles is considered to reflect ecology and feeding habits (Busbey, 1994; Bro$\mathrm{chu}, 2001)$ and it is reasonable to assume the same was true of choristoderes. Busbey (1994) characterised crocodile skulls as platyrostral (dorsoventrally compressed) or oreinirostral (mediolaterally compressed) according to rostrum width/depth indices, and as short, medium or long according to the length of the rostrum relative to overall skull length. However, the same metrics do not work well for choristoderes because their skulls and rostra are more dorsoventrally flattened and because the postorbital skull proportions of crocodiles and choristoderes are very different. Thus plotting choristoderes onto Busbey's (1994) graph of crocodilian skull morphotypes (Fig. 7) fails, for example, to identify neochoristoderes as longirostrine (despite widespread use of the gavial as a functional analogue for Champsosaurus: e.g. Erickson, 1972, Evans and Hecht, 1993), and the clearly longirostrine Simoedosaurus emerges within the brevirostrine crocodile cluster. Different parameters need to be identified for meaningful comparison. Nonetheless, if crocodiles and choristoderes were interacting ecologically, one would predict that, in any one locality, the choristodere rostral shape would not be replicated by any of the co-occurring crocodiles. A comparison of rostral shapes (Fig. 6) mainly confirms this prediction, although the comparisons are not statistical. There are two horizons (Upper Cretaceous Hell Creek Formation, Palaeocene Hannut Formation) where crocodiles and choristoderes with slender rostra are found together, but there is reasonable evidence to suggest they did not live together (see above). Indeed, longirostrine crocodilians were primarily in coastal and marine environments, leaving a vacant niche in freshwater ecosystems that neochoristoderes occupied.

In this section, we have focused on the possible ecological relationship between choristoderes and crocodiles because neochoristoderes are most frequently compared 


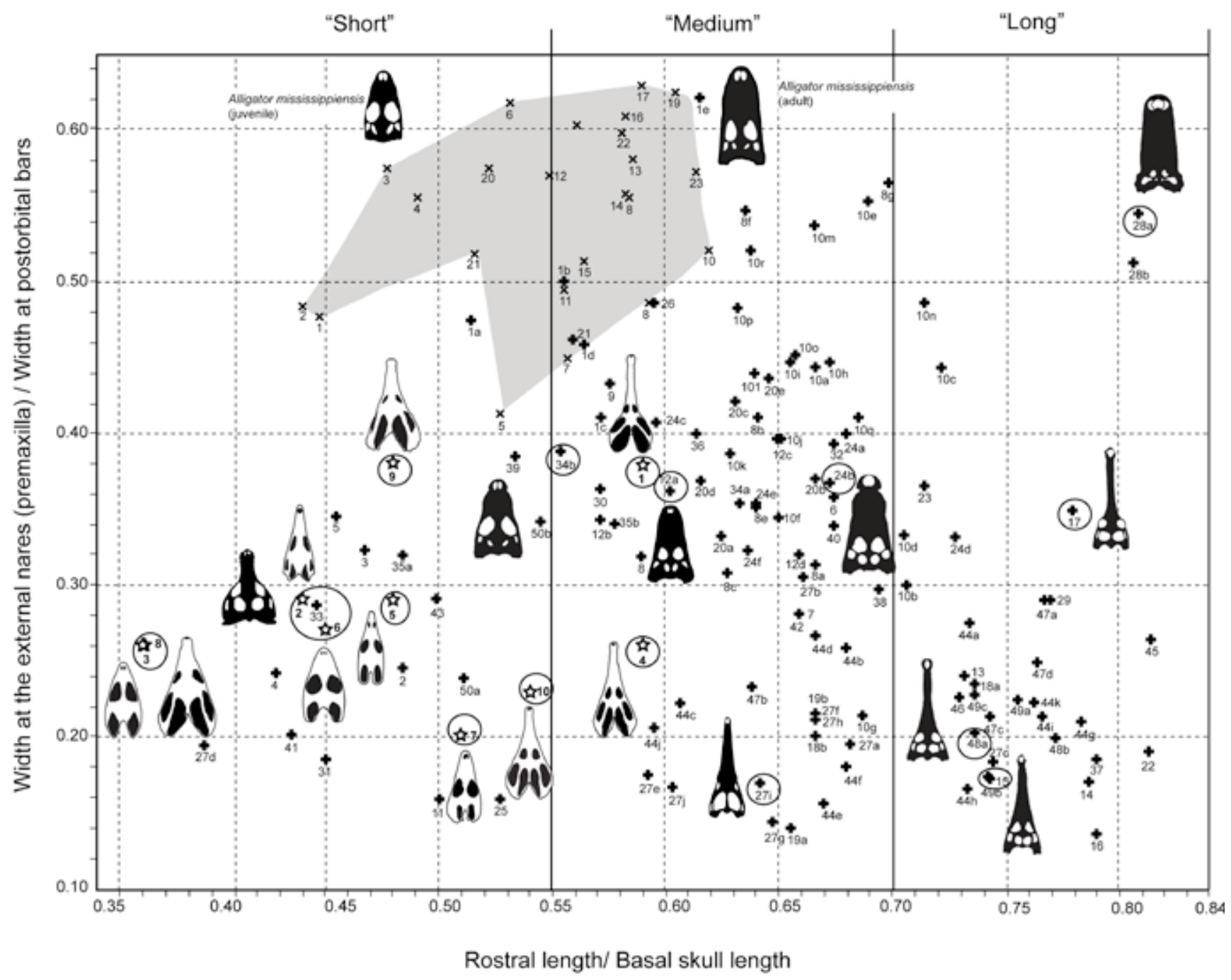

Fig. 7.- Plot comparing rostral width and length for choristoderes and crocodiles (based on Busbey 1994, figure 10. 2). Choristoderes are indicated by numbered stars: 1, Champsosaurus gigas (Erickson, 1985); 2, Cteniogenys sp. (Evans 1990); 3, Hyphalosaurus sp. (IVPP V 14560); 4, Ikechosaurus pijiagouensis (Liu, 2004); 5, Lazarussuchus inexpextatus (Hecht, 1992); 6, Philydrosaurus proseilus (Gao et al., 2007); Simoedosaurus dakotensis (Erickson, 1987); Simoedosaurus sp. (SMNS 59026); 10, Tchoiria namsarai (undescribed Hayashibara specimens). The area marked in grey shows ontogenetic variation for Alligator mississippiensis, specimens marked by x. Other crocodiles are marked with crosses. Encircled numbers correspond to illustrated skull silhouettes, choristoderes in white and crocodiles in black silhouettes. The illustrated crocodiles are: 12a, Diplocynodon darwini; 15, Eosuchus lerichei; 17, Gavialis lewisi; 24b, Leidyosuchus canadensis; 27i, Metriorhynchus superciliosum; 28a, Mourasuchus amazonensis; 33 Orthosuchus stormbergi; 34b, Osteolaemus tetraspis; 48a, Thoracosaurus macrorhynchus. For a key to the other crocodiles, see Busbey (1994).

Fig. 7.- Gráfica donde se compara la anchura y lontud rostral en coristoderos y en cocodrilos (basado en Busbey 1994, figure 10. 2). Los coristoderos se indican mediante estrellas numeradas: 1, Champsosaurus gigas (Erickson, 1985); 2, Cteniogenys sp. (Evans 1990); 3, Hyphalosaurus sp. (IVPP V 14560); 4, Ikechosaurus pijiagouensis (Liu, 2004); 5, Lazarussuchus inexpextatus (Hecht, 1992); 6, Philydrosaurus proseilus (Gao et al., 2007); Simoedosaurus dakotensis (Erickson, 1987); Simoedosaurus sp. (SMNS 59026); 10, Tchoiria namsarai (especímenes no descritos de Hayashibara). El area marcada en gris muestra la variación ontogenética de Alligator mississippiensis, especímenes marcados con una x. Los demás cocodrilos se han marcado con cruces. Los números incluidos en un círculo corresponden a las siluetas de los cráneos ilustrados (en blanco corsitoderos, en negro cocodrilos). Los cocodrilos ilustrados son: 12a, Diplocynodon darwini; 15, Eosuchus lerichei; 17, Gavialis lewisi; 24b, Leidyosuchus canadensis; 27i, Metriorhynchus superciliosum; 28a, Mourasuchus amazonensis; 33 Orthosuchus stormbergi; 34b, Osteolaemus tetraspis; 48a, Thoracosaurus macrorhynchus. La clave para los restantes cocodrilos en Busbey (1994).

with gavialids in terms of lifestyle. However, understanding these potential interactions requires a fuller knowledge of choristoderan diet and lifestyle, which ongoing studies of the gut contents, dentition and skull morphology should help to elaborate.

\subsection{Choristoderan evolution and palaeobiogeography}

Although the phylogenetic position of choristoderes within Diapsida has yet to be resolved, all of the proposed positions (stem-neodiapsid, basal archosauromorph, sau- 
ropterygian relative etc) would predict an origin in the Late Permian or earliest Triassic. As yet, there is no record for that period and thus any discussion of early choristoderan evolution is speculative. Nonetheless, the earliest taxon that can be attributed to the group with confidence is the Middle-Late Jurassic Cteniogenys as well as similar, but currently indeterminate material from Central Asia. These early choristoderes are small and constitute part of a freshwater aquatic assemblage that continues almost unchanged (except for the representative genera) until the Miocene. In most cases, these small Jurassic choristoderes are found in swampy or lagoonal freshwater coastal environments. The absence of Permian or Triassic representatives almost certainly reflects the paucity of suitable depositional environments, as evidenced by the absence, or near absence, of Triassic frogs, salamanders, lizards, turtles, and albanerpetontids which typically occur with choristoderes in younger deposits. Choristoderes presumably evolved from small terrestrialamphibious Permian diapsids within the northern part of Pangea. The Permo-Triassic arid belt may have represented a significant barrier as they did not reach southern Pangea (and have never been recorded from Gondwana).

Small choristoderes had spread across Laurasia by the end of the Jurassic but then disappeared from the Euramerican record (the enigmatic fragments from the Late Cretaceous of Alberta excepted). In Eastern Asia, isolated from the rest of Laurasia in the Middle Jurassic-Lower Cretaceous, the group radiated and diversified, giving rise to the larger neochoristoderes, as represented by Tchoiria and Ikechosaurus. The apparent absence of aquatic neosuchian crocodiles in these continental localities/ecosystems may well have been crucial to this morphological diversification and almost certainly facilitated the evolution of the large gavial-like choristoderan ecomorphs. In the Lower Cretaceous, Eastern Asia established a connection with western North America, and this may be the route through which neochoristoderes (first recorded in the Barremian of Utah, Britt et al., 2006) entered North America. Neochoristoderes then seem to have established themselves across Laurasia, successfully sharing freshwater ecosystems with eusuchian crocodiles as long as resources were sufficient and there were no direct longirostrine crocodilian competitors. The possible role of crocodiles in the ultimate extinction of neochoristoderes remains unknown, given the accompanying climatic changes at the Paleocene-Eocene transition, but may be clarified with a greater understanding of choristoderan functional anatomy and thus possible areas of niche overlap.

The reappearance of non-neochoristoderes in the Paleogene of Europe was surprising, as reflected by the name
Lazarussuchus (Hecht, 1992). Whether this genus is a relict of the original Jurassic distribution, or was derived from an Asian lineage that reinvaded Euramerica when contact was reestablished, is currently uncertain, as the phylogenetic position of Lazarussuchus is not fully resolved (e.g. Matsumoto et al., 2007, 2009; Skutschas, 2008). Material of earlier choristoderes would shed light on this. Finally, although the last record of choristoderes is currently from the lower Miocene, the group may have survived longer at other localities. Albanerpetontid amphibians provide a parallel case. They were long thought to have become extinct in the Miocene (e.g. Estes, 1981) but have since been found in the Lower Pliocene of Hungary (Venczel and Gardner, 2005) and the Late Pliocene of Italy (Delfino and Sala, 2007). A dedicated reexamination of all suitable Mesozoic, Paleogene and Neogene small vertebrate assemblages throughout Laurasia is needed to provide more confident presence/absence data for these periods so that the history, palaeobiogeography, phylogeny, and ecology of this enigmatic reptilian group can be better understood.

\section{Conclusions}

Choristoderes are generally found in association with a diverse freshwater vertebrate assemblage, in warm temperate environments across Laurasia.

Allowing for preservational and collecting biases, much of the Jurassic choristodere record, and at least some of that for Late Cretaceous and Paleocene neochoristoderes, comes from low-lying depositional freshwater environments close to the coast. In the Lower Cretaceous of Asia and the Paleogene of Europe, they appear to have been in more continental water bodies.

Choristoderes typically co-occur with crocodiles, but were morphologically most diverse in Asian deposits where aquatic crocodiles were absent.

Neochoristoderes apparently evolved in an isolated Jurassic-Cretaceous Asia, within freshwater environments where the absence (or rarity) of crocodiles left a vacant large longirostrine piscivore niche to be exploited.

Where large neochoristoderes and crocodiles co-occur, the former are typically the only reptiles with strongly longirostrine skulls, suggesting a degree of niche partitioning, aided by the absence of longirostrine crocodiles from Mesozoic and Paleogene freshwater deposits.

\section{Acknowledgements}

We would like to thank Dr Alexander O. Averianov (Zoological Institute, Russian Academy of Sciences, St 
Peterburg), Ms Chamero Beatriz (Universidad Autonoma Madrid), Dr Marco Branadlise de Andrade (University of Bristol, UK), Dr Shin-ichi Fujiwara (University of Tokyo, Japan), Dr Pavel Skutchas (St Petersburg University, Russia), Dr Denise Sigogneau-Russell (Paris), Dr Komsorn Lauprasert (Mahasarakham University, Thailand), Dr Harufumi Nishida (Chuo University, Japan), and Dr Xiao-Chun $\mathrm{Wu}$ (Canadian Museum of Nature, Ottawa, Canada) for creative suggestions and information for this study; as well as Dr Bruce Erickson and Ms Jackie Hoff (Science Museum Minnesota), Drs Don Brinkman and Don Henderson (Royal Tyrrell Museum), Dr. Bernard Battail, (Muséum National d'Histoire Naturelle), Dr Rainer Schoch (Staatliches Museum für Naturkunde, Germany), and Dr Yuan Wang (Institute of Vertebrate Paleontology and Paleoanthropology, Beijing, China) for access to specimens. Our particular thanks to Drs Angela D. Buscalioni (Universidad Autónoma Madrid) and Eric Buffetaut (CNRS, France) for discussion of crocodilian phylogeny, palaeoecology and distribution. RM acknowledges funding from a $10^{\text {th }}$ MTE Student Travel Scholarship; the Palaeontological Association, England; the University of London Central Research Fund; and a Synthesys grant (MNHM, Paris).

\section{References}

Averianov, A. O. (2005): The first choristoderes (Diapsida, Choristodera) from the Paleogene of Asia. Paleontological Journal, 39: 79-84.

Averianov, A. O., Martin, T., Evans, S. E., Bakirov, A. A. (2006): First Jurassic Choristodera from Asia. Naturwissenschaften, 93: 46-50. http://dx.doi.org/10.1007/s00114-005-0061-2

Averianov, A.O., Skutchas, P. (2000): A eutherian mammal from the Early Cretaceous of Russia and biostratigraphy of the Asian Early Cretaceous vertebrate assemblages. Lethaia, 33: 330-340.

Azuma, Y. (2003): Early Cretaceous vertebrate remains from Katsuyama City, Fukui Prefecture, Japan, Memoir of the Fukui Prefectural Dinosaur Museum, 2: 17-21.

Bartels, W. S. (1983): A transitional Paleocene-Eocene reptile fauna from the Bighorn Basin, Wyoming. Herpetologica, 39: 359-374.

Bartels, W. S. (1984): Osteology and systematic affinities of the horned alligator Ceratosuchus (Reptilia, Crocodilia). Journal of Paleontology, 58: 1347-1353.

Bellairs, A. d'A. (1969): The life of reptiles. Vol. II. Weidenfeld and Nicolson, London.

Böhme, M. (2008): Ectothermic vertebrates (Teleostei, Allocaudata, Urodela, Anura, Testudines, Choristodera, Crocodylia, Squamata) from the Upper Oligocene of Oberleichtersbach (Northern Bavaria, Germany). Courier Forschungsinstitut Senckenberg, 260: 161-183.

Brochu, C.A. (1997): A review of "Leidyosuchus" (Crocodyliformes, Eusuchia) from the Cretaceous through Eocene of
North America. Journal of Vertebrate Paleontology, 17: 679-97. http://dx.doi.org/10.1080/02724634.1997.10011017

Brochu, C.A. (2001): Crocodylian snouts in space and time: phylogenetic approaches toward adaptive radiation. American Zoologist, 41: 564- 585. http://dx.doi.org/10.1093/icb/41.3.564

Brinkman, D. B. (1990): Paleoecology of the Judith River Formation (Campanian) of Dinosaur Provincial Park, Alberta, Canada: evidence from vertebrate microfossil localities. Palaeogeography, Palaeoclimatology, Palaeoecology, 78: 37-54. http:// dx.doi.org/10.1016/0031-0182(90)90203-J

Brinkman, D. B., Dong, Z. M, (1993): A new material of Ikechosaurus sunailinae (Reptilia: Choristodera) from the Early Cretaceous Langhongdong Formation, Ordos Basin, Inner Mongolia, and the interrelationships of the genus. Canadian Journal of Earth Sciences, 30: 2153-2162.

Brinkmann, W. (1989): Vorläufige Mitteilung über die Krokodilier-Faunen aus dem Ober-Jura (Kimmeridgium) der Kohlegrube Guimarota, bei Leiria (Portugal) und der Unter-Kreide (Barremium) von Uña (Provinz Cuenca, Spanien), Documenta Naturae, 56: 1-28.

Britt, B. B., Scheetz, R. D., Brinkman, D. B., Eberth, D. A. (2006): A Barremian neochoristodere from the Cedar Mountain Formation, Utah, U.S.A. Journal of Vertebrate Paleontology, 26:1005-1008.

Britt, B. B., Eberth, D. A., Scheetz, R. D., Greenhalgh, B. W., Stadtman K. L. (2009): Taphonomy of debris-flow hosted dinosaur bonebeds at Dalton Wells, Utah (Lower Cretaceous, Cedar Mountain Formation, USA). Palaeogeography, Palaeoclimatology, Palaeoecology, 280: 1-22. http://dx.doi.org/10.1016/j. palaeo.2009.06.004

Brown, B. (1905): The osteology of Champsosaurus Cope. American Museum of Natural History, Memoirs, 9: 1-26.

Buffetaut, E. (1979): Revision der Crocodylia (Reptilia) aus der Gosau- Schichten (Ober-Kreide) von Österreich. Beiträge zur Paläontologie von Österreich, 6: 89-105.

Buffetaut, E. (1989): Erster Nachweis von Choristodera (Reptilia, Diapsida) in der Oberkreide Europas: Champsosaurierwirbel aus den Gosau-Schichten (Campan) Niederösterreichs. Sitzungberichte Österreichische Akademie der Wissenschaften, Mathematisch-naturwissenschaftliche Klasse, 197: 389-394.

Busbey, A. B. (1994): The structural consequences of skull flattening in crocodilians. In: J. J. Thomason (ed.), Functional morphology in vertebrate paleontology, Cambridge University Press, New York: 173-192.

Buscalioni A. D., Fregenal-Martinez, M. A. (2006): Archosaurian size bias in Jurassic and Cretaceous freshwater ecosystems. In: P. Barrett, S.E. Evans (eds.) 9th Mesozoic Terrestrial Ecosystems and Biota, Manchester, Short papers: 9-12.

Buscalioni, A. D., Pérez-Moreno, B. P., Sanz, J. L. (2003): Pattern of biotic replacement in modern crocodiles during the Late Cretaceous. Coloquios de Paleontología, Homenaje a Remmert Daams: 77-94.

Charig, A. J., Krebs, B., Sues, H.-D., Westphal, F. (1976): Thecodontia. Encyclopedia of Paleoherpetology, Vol. 13. Gustav Fischer Verlag, New York and Stuttgart: 137 p.

Chure, D., Evans, S. E. (1998): A new occurence of Cteniogenys, with comments on its distribution and abundance. Modern Geology, 23: 49-55. 
Chure, D. J., Carpenter, K., Litwin, R., Hasiotis, S., Evanoff, E. (1998): Appendix. The fauna and flora of the Morrison Formation. In: K. Carpenter, D.J. Chure, J. I. Kirkland (eds.), The Upper Jurassic Morrison Formation: An Interdisciplinary Study 23/1-4, Modern Geology: 507-537.

Cifelli, R. L., Nydam, R. L., Eaton, J. G., Gardner, J. D., Kirkland, J. I. (1999a): Vertebrate faunas of the North Horn Formation (Upper Cretaceous-Lower Paleocene), Emery and Sanpete Counties, Utah. In: D. Gillette (ed.), Vertebrate Paleontology in Utah, Utah Geological Survey, Miscellaneous Publication 99-1: 377-388.

Cifelli, R. L., Nydam, R. L., Gardner, J. D., Weil, A., Eaton, J. G., Kirkland J. I., Madsen, S. K. (1999b): Medial Cretaceous vertebrates from the Cedar Mountain Formation, Emery County, Utah: the Mussentuchit Local Fauna. In: D. Gillette (ed.), Vertebrate Paleontology in Utah, Utah Geological Survey, Miscellaneous Publication 99-1: 219-242.

Clark, J. M. (1994): Patterns of evolution in Mesozoic Crocodyliformes. In: N. Fraser, H-D. Sues (eds.), In the Shadow of the Dinosaurs: Early Mesozoic Tetrapods, Cambridge University Press, Cambridge: 84-97.

Cope, E. D. (1876): On some extinct reptiles and Batrachia from the Judith River and Fox Hills beds of Montana. Proceedings of the National Academy of Sciences 28: 340-359.

Company, J. Suberbiola, X. P., Ruiz-Omenaca, J. I., Buscalioni, A. D. (2005): A new species of Doratodon (Crocodyliformes: Ziphosuchia) from the Late Cretaceous of Spain. Journal of Vertebrate Paleontology, 25: 343-353.

De Braga, M., Rieppel, O. (1997): Reptile phylogeny and the interrelationships of turtles. Zoological Journal of the Linnean Society, 120: 281-354.

Delfino, M., Sala, B. (2007): Late Pliocene Albanerpetontidae (Lissamphibia) from Italy. Journal of Vertebrate Paleontology, 27: 716-719.

Delfino, M., Piras, P. Smith, T. (2005): Anatomy and phylogeny of the gavialoid Eosuchus lerichei from the Paleocene of Europe. Acta Palaeontologica Polonica, 50: 565-580.

DeMar, D., Breithaupt, B. (2008): Terrestrial and aquatic vertebrate paleocommunities of the Mesaverde Formation (Upper Cretaceous, Campanian) of the Milk River and Bighorn Basins, Wyoming, USA. In: J. Sankey, S. Baszio (eds.), Vertebrate Microfossil Assemblages, Indiana University Press, Bloomington and Indianapolis: 78-103.

Dodson, P. (1975): Functional and ecological significance of relative growth in Alligator. Journal of Zoology, 175: 315-355. http://dx.doi.org/10.1111/j.1469-7998.1975.tb01405.x

Dilkes, D. W. (1998): The Early Triassic rhynchosaur Mesosuchus browni and the interrelationships of basal archosauromorph reptiles. Philosophical Transactions of the Royal Society of London, B353: 501-541. http://dx.doi.org/10.1098/rstb.1998.0225

Efimov, M. B. (1975): Champsosaurs from the Lower Cretaceous of Mongolia [in Russian]. Trudy Sovmestnoi SovetskoMongol'skoi Paleontologicheskoi Ekspeditsii, 2: 84-93.

Efimov, M. B. (1979). Tchoiria (Champsosauridae) from the Early Cretaceous of Khamaryn Khural, MNR [in Russian]. Trudy Sovmestnoi Sovetsko-Mongol'skoi Paleontologicheskoi Ekspeditsii, 8: 56-57.

Efimov, M. B., Storrs, G. W. (2000): Choristodera from the Lower Cretaceous of northern Asia. In: M. J. Benton, M. A. Shishkin,
D. M. Unwin, E. N. Kurochkin (eds.), The age of dinosaurs in Russia and Mongolia: Cambridge University Press, Cambridge: 390-401.

Endo, R. (1940): A new genus of Thecodontia from the Lycoptera beds in Manchoukuo. Bulletin of the Central National Museum of Manchoukuo, 2: 1-14.

Erickson, B. R. (1972): The lepidosaurian reptile Champsosaurus in North America. The Science Museum of Minnesota, Monograph (Paleontology), 1: 1-91.

Erickson, B. R. (1982): Wannaganosuchus, a new alligator from the Paleocene of North America. Journal of Paleontology, 56: 492-506.

Erickson, B. R. (1985): Aspects of some anatomical structures of Champsosaurus Cope (Reptilia: Eosuchia). Journal of Vertebrate Paleontology, 5: 111-127. http://dx.doi.org/10.1080/027 24634.1985.10011849

Erickson, B. R. (1987): Simoedosaurus dakotensis, new species, a diapsid reptile (Archosauromorpha: Choristodera) from the Paleocene of North America. Journal of Vertebrate Paleontology, 7: 237-251. http://dx.doi.org/10.1080/02724634.1987.1 $\underline{0011658}$

Erickson, B. R. (1999): Fossil Lake Wannagan (Paleocene: Tiffanian) Billings County, North Dakota. North Dakota Geological Survey, Miscellaneous Series number, 87: 1-9.

Estes, R. (1981): Gymnophiona, Caudata. Handbuch der Paläoherpetologie part 2. Gustav Fischer Verlag, New York: 115 p.

Evans, S. E. (1988): The early history and relationships of the Diapsida. In: M.J. Benton (ed.), The phylogeny and classification of the Tetrapoda, 1. Amphibians, reptiles, birds: Clarendon Press, Oxford: 221-260.

Evans, S. E. (1989): New material of Cteniogenys (Reptilia: Diapsida) and a reassessment of the systematic position of the genus. Neues Jahrbuch für Geologische und Palaontologische, Abhundlungen, 181: 577-589.

Evans, S. E. (1990): The skull of Cteniogenys, a choristodere (Reptilia: Archosauromorpha) from the Middle Jurassic of Oxfordshire. Zoological Journal of the Linnean Society, 99: 205237.http://dx.doi.org/10.1111/j.1096-3642.1990.tb00561.x

Evans, S. E., Hecht, M. K. (1993): A history of an extinct reptilian clade, the Choristodera: longevity, Lazarus-taxa, and the fossil record. Evolutionary Biology, 27: 323-338.

Evans, S. E., Klembara, J. (2005): A choristoderan reptile (Reptilia: Diapsida) from the Lower Miocene of Northwest Bohemia (Czech Republic). Journal of Vertebrate Paleontology, 25: 171184.

Evans, S. E., Manabe, M. (1999): A choristoderan reptile from the Lower Cretaceous of Japan. Special Papers in Paleontology, 60: 101-119.

Evans, S. E., Milner, A. R. (1994): Microvertebrate faunas from the Middle Jurassic of Britain. In: N. Fraser, H. D. Sues (eds.), In the Shadow of the Dinosaurs: Early Mesozoic Tetrapods, Cambridge University Press: 303-321.

Evans, S. E., Waldman, M. (1996): Reptiles and amphibians from the Middle Jurassic (Bathonian) of Skye, Scotland. In: M. Morales (ed.), The Continental Jurassic, Museum of Northern Arizona Bulletin, 60: 219-226.

Fejfar, O., Schleich, H. H. (1994): Ein Chamaeleonfund aus dem Orleanian des Braunkohle-Tagebaus Merkur-Nord (Nord-böhmen). Courier Forschung-Institut Senckenberg, 173: 167-173. 
Foster, J. R. Trujillo, K. C. (2000): New occurrences of Cteniogenys (Reptilia, Choristodera) in the Late Jurassic of Wyoming and South Dakota. Brigham Young University Geology Studies, 45: 11-18.

Fujita A, M. (2003): Geological age and correlation ofthe vertebrate-bearing horizons in the Tetori Group. Memoir of the Fukui Prefectural Dinosaur Museum, 2: 3-14.

Gao C. L., Lu, J. C., Liu, J. Y., Ji, Q. (2005): New Choristodera from the Lower Cretaceous Jiufotang Formation in Chaoyang area, Liaoning China (in Chinese). Geological Review, 51: 694697.

Gao, K. Q., Brinkman, D. B. (2005): Choristoderes from the Park and its vicinity. In: Currie, P. J., Koppelhus, E. B. (eds.), Dinosaur Provincial Park, A Spectacular Ancient Ecosystem Revealed. Indiana University Press, Indianapolis: 221-234.

Gao, K., Evans, S. E., Ji, Q., Norell, M., Ji, S. (2000): Exceptional fossil material of a semi-aquatic reptile from China: the resolution of an enigma. Journal of Vertebrate Paleontology, 20:417421.

Gao, K., Fox, R. C. (1998): New choristoderes (Reptilia: Diapsida) from the Upper Cretaceous and Palaeocene, Alberta and Saskatchewan, Canada, and phylogenetic relationships of the Choristodera. Zoological Journal of the Linnean Society, 124: 303353. http://dx.doi.org/10.1111/j.1096-3642.1998.tb00580.x

Gao, K., Fox, R. C. (2005): A new choristodere (Reptilia: Diapsida) from the Lower Cretaceous of western Liaoning Province, China, and phylogenetic relationships of Monjurosuchidae. Zoological Journal of the Linnean Society, 145: 427-444. http:// dx.doi.org/10.1111/j.1096-3642.2005.00191.x

Gao, K., Ksepka, D. T., Hou, L. H., Ye, D., Hu, D. (2007): Cranial morphology of an Early Cretaceous monjurosuchid (Reptilia: Diapsida) from Liaoning Province of China and evolution of the choristoderan palate. Historical Biology, 19: 215-224. http:// dx.doi.org/10.1080/08912960601106391

Gao, K., Ksepka, D. T. (2008): Osteology and taxonomic revision of Hyphalosaurus (Diapsida: Choristodera) from the Lower Cretaceous of Liaoning, China. Journal of Anatomy, 212: 747768. http://dx.doi.org/10.1111/j.1469-7580.2008.00907.x

Gao, K., Tang, Z., Wang, X. (1999): A long-necked diapsid reptile from the Upper Jurassic/ Lower Cretaceous of Liaoning Province, north-eastern China. Vertebrata PalAsiatica, 37:1-8.

Garrison, J. R. Jr., Brinkman, D., Nichols, D. J., Layer, P., Burge, D., Thayne, D. (2007): A multidisciplinary study of the Lower Cretaceous Cedar Mountain Formation, Mussentuchit Wash, Utah - a determination of the paleoenvironment and paleoecology of the Eolambia caroljonesa dinosaur quarry. Cretaceous Research, 28: 461-494.

Gauthier, J. A., Kluge, A., Rowe, T. (1988): Amniote phylogeny and the importance of fossils. Cladistics, 4: 105-209. http:// dx.doi.org/10.1111/j.1096-0031.1988.tb00514.x

Gervais, P. (1877): Enumération de quelques ossements d'animaux vertébrés recueillis aux environs de Reims par M. Lemoine. Journal de Zoologie, 6: 74-79.

Gilmore, C.W. (1928): Fossil lizards of North America. Memoirs of the National Academy of Science, 22:1-169.

Gingerich, P. D. (2000): Paleocene/Eocene boundary and continental vertebrate faunas of Europe and North America. Geologiska Föreningens Förhandlingar, Geologi- cal Society of Sweden, Uppsala, 122: 57-59. http://dx.doi. org/10.1080/11035890001221057

Göhlich, U. B., Chiappe, L. M., Clark, J. M., Sues, H-D. (2005): The systematic position of the Late Jurassic alleged dinosaur Macelognathus (Crocodylomorpha: Sphenosuchia). Canadian Journal of Earth Sciences, 42: 307-321. http://dx.doi. org/10.1139/e05-005

Grigorescu, D. Venczel, M., Csiki, Z., Limberea, R. (1999): New latest Cretaceous microvertebrate fossil assemblages from the Hateg Basin (Romania). Geologie en Mijnbouw, 78: 301-314. http://dx.doi.org/10.1023/A:1003890913328

Gunnell, G. F., Bartels, W. S., Gingerich, P. D. (1993): PaleoceneEocene boundary in continental North America: biostratigraphy and geochronology, northern Bighorn Basin, Wyoming. New Mexico Museum of Natural History and Science Bulletin, 2: 137-144.

Hecht, M. K. (1992): A new choristodere (Reptilia, Diapsida) from the Oligocene of France: an example of the Lazarus effect. Geobios, 25:115-131. http://dx.doi.org/10.1016/S0016$\underline{6995(09) 90041-9}$

Herman, A. B., Kvacek, J. (2007): Early Campanian Grunbach flora of Austria. Paleontological Journal, 41: 1068-1076. http:// dx.doi.org/10.1134/S0031030107110068

Hups, K. M., Foster, J., Lockley, M. (2005): A partial skeleton of Goniopholis from the Brushy Basin Member of the Morrison Formation. Geological Society of America Abstracts, Rocky Mountain Section - 57th Annual Meeting (May 23-25, 2005), Paper No. 7-10.

Ji, Q., Ji, S., Cheng, Y., You, H., Lu, J., Yuan, C. (2004): The first fossil soft-shell eggs with embryos from Late Mesozoic Jehol Biota of western Liaoning, China. Acta Geoscientica Sinica, 25: 275-285.

Jouve, S., Bardet, N. Jalil, N.E., Pereda, X., Suberbiola, Bouya, B., Amaghzaz, M. (2008): The oldest African crocodylian: phylogeny, paleobiogeography, and differential survivorship of marine reptiles through the Cretaceous-Tertiary boundary. Journal Vertebrate Paleontology, 28: 409-421. http://dx.doi. org/10.1671/0272-4634(2008)28[409:TOACPP]2.0.CO;2

Kimura, T. (1979): Late Mesozoic palaeofloristic provinces in East Asia. Proceedings of the Japan Academy, 55: 425-430. http:// dx.doi.org/10.2183/pjab.55.425

Kirkland, J. I., Cifelli, R. L., Britt, B. B., Burge, D. L., DeCourten, F. L., Eaton, J. G., Parrish, J. M. (1999): Distribution of vertebrate faunas in the Cedar Mountain Formation, East-Central Utah. In: D.D. Gillette (ed.), Vertebrate Paleontology in Utah. Miscellaneous Publication 99-1, Utah Geological Survey: 201217.

Krebs, B. (1967): Der Jura-Krokodilier Machimosaurus H. v. Meyer. Paläontologische Zeitschrift, 41: 46-59.

Kusuhashi, N. (2008): Early Cretaceous multituberculate mammals from the Kuwajima Formation (Tetori Group), central Japan. Acta Palaeontologica Polonica, 53: 379-390. http:// dx.doi.org/10.4202/app.2008.0302

Lauprasert, K. Cuny, G. Thirakhupt K. and Suteethorn V. (2009): Khoratosuchus jintasakuli gen. et sp. nov., an advanced neosuchian crocodyliform from the Early Cretaceous (Aptian-Albian) of NE Thailand. Geological Society, London, Special Publications, 315: 175-187. http://dx.doi.org/10.1144/SP315.13 
Leidy, J. (1852): Vice-President Bridges in the chair. Academy of Natural Sciences of Philadelphia, 6: 34-36.

Liu, J. (2004): A nearly complete skeleton of Ikechosaurus pijiagouensis sp. nov. (Reptilia: Choristodera) from the Jiufotang Formation (Lower Cretaceous) of Liaoning, China. Vertebrata PalAsiatica, 42: 120-129.

Lucas, S. G., Estep, J. W. (2000): Osteology of Allognathosuchus mooki Simpson, a Paleocene crocodilian from the San Juan Basin, New México, and the monophyly of Allognathosuchus. New México Museum of Natural History and Science Bulletin, 16:155-168.

Luiselli, L., Akani, G. C., Capizzi, D. (1999): Is there any interspecific competition between dwarf crocodiles (Osteolaemus tetraspis) and Nile monitors (Varanus niloticus ornatus) in the swamps of central Africa? A study from south-eastern Nigeria. Journal of Zoology, London, 247: 127-131. http://dx.doi. org/10.1017/S0952836999001132

Marsh, O.C. (1870): Notice of a new species of gavial from the Eocene of New Jersey. American Journal of Science, 50: 2-3.

Markwick, P. J. (1998): Fossil crocodiles as indicators of Late Cretaceous and Cenozoic climates: implications for using palaeontological data in reconstructing palaeoclimate. Palaeogeography, Palaeoclimatology, Palaeoecology, 137: 205-271.

Martin, J. E. (2008): New material of the alleged oldest Diplocynodon from the Late Paleocene of northeastern France: revision and implications for crocodylian dispersal. Vertebrate Palaeontology and Comparative Anatomy, the Annual Symposium (September 1-6, 2008).

Martin, T., Krebs, B. (2000): Guimarota. A Jurassic Ecosystem. Pfeil, München: 155 p.

Matsumoto, R., Evans, S.E., Manabe, M. (2007): The choristoderan reptile Monjurosuchus from the Early Cretaceous of Japan. Acta Palaeontotogica Polonica, 52: 329-350.

Matsumoto, R., Suzuki, S., Tsogtbaatar K., Evans S.E. (2009): New material of the enigmatic reptile Khurendukhosaurus (Diapsida: Choristodera) from Mongolia. Naturwissenschaften, 96 : 233-242. http://dx.doi.org/10.1007/s00114-008-0469-6

Meesook, A. (2000): 3. In: H. Okada, N. Mateer (eds), Cretaceous Environments of Asia. Elsevier Science BV: 207-223. http:// dx.doi.org/10.1016/S0920-5446(00)80034-5

Morlo, M., Schall, S., Mayr G., Seiffert C. (2004): An annotated taxonomic list of the Middle Eocene (MP 11) Vertebrata of Messel. Courier Forschungsinstitut Senckenberg, 252: 79-93.

Müller, J. (2004): The relationships among diapsid reptiles and the influence of taxon selection. In: G. Arratia, R. Cloutier, V.H. Wilson (eds.), Recent advances in the origin and early radiation of vertebrates. Pfeil, München: 379-408.

Norell, M.A., Clark, J.M., Hutchison, J. H. (1994): The Late Cretaceous alligatoroid Brachychampsa montana (Crocodylia): New material and putative relationships. American Museum Novitates, 3116: 1-26.

Ortega, F. Sanz, J.L., Barbadillo, L., Buscalioni, A.C., Diéguez, C., Evans, S.E., Fregenal-Martínez, M.A., Fuente, M., Madero, J., Martín-Closas, C., Martínez-Delclós, X., Meléndez N., Moratalla, J.J., Pérez-Moreno, P., Pinardo-Moya, E., Poyato-Aroza, F.J., Lázaro, JR., Sanchiz, B., Wenz, S. (1999): El Yacimiento de Las Hoyas (la Cierva, Cuenca): un Konservat-lagerstätte del Cretácico inferior. In: Aguirre, E., Rábano, I. (eds), La Huella del Pasado: Fósiles de Castilla-La Mancha. (Patrimonio Histó-
rico-Arqueología de Castilla-La Mancha) Junta de comunidades de Castilla-La Mancha, Toledo: 197 p.

Pearson, D. A, Schaefer, T., Johnson, K. R., Nichols, D. J, Hunter, J. P. (2002): Vertebrate biostratigraphy of the Hell Creek Formation in southwestern North Dakota and northwestern South Dakota. In: J. H. Hartman, K. R. Johnson, D. J. Nichols (eds.), The Hell Creek Formation and the Cretaceous-Tertiary Boundary in the Northern Great Plains: An Integrated Continental Record of the End of the Cretaceous, Geological Society of America Special Paper, Volume 361, Boulder (CO): 145-167.

Piras, P., Buscalioni, A. D. (2006): Diplocynodon muelleri comb. nov., an Oligocene diplocynodontine alligatoroid from Catalonia (Ebro Basin, Lleida Province, Spain). Journal of Vertebrate Paleontology, 26: 608-620. http://dx.doi.org/10.1671/02724634(2006)26[608:DMCNAO]2.0.CO;2

Prasad, G. V. R., de Lapparent de Broin, F. (2002): Late Cretaceous crocodile remains from Naskal (India): comparisons and biogeographic affinities. Annales de Paléontologie, 88: 19-71.

Rieppel, O. (1998): The status of the sauropterygian reptile genera Ceresiosaurus, Lariosaurus, and Silvestrosaurus from the Middle Triassic of Europe. Fieldiana, 38:1-46.

Russell, L. S. (1956): The Cretaceous reptile Champsosaurus natator Parks. National Museum of Canada, Bulletin, 145: 1-51.

Russell, L. S. (1974): Fauna and correlation of the Ravenscrag Formation (Paleocene) of southwestern Saskatchewan. Life Sciences Contributions, Royal Ontario Museum, 102: 1-53.

Russell, D. E., de Broin, F., Galoyer, A., Gaudant, J., Gingerich, P. D., Rage, J.-C. (1990): Les vertébrés du Sparnacien de Meudon, France. Comptes Rendus d'Academie des Sciences, Paris Sér.II, 307: 429-433.

Sachs, S., Hornung, J. J. (2006): Juvenile ornithopod (Dinosauria: Rhabdodontidae) remains from the Upper Cretaceous (Lower Campanian, Gosau Group) of Muthmannsdorf (Lower Austria). Geobios, 39: 415-425. http://dx.doi.org/10.1016/j.geobios.2005.01.003

Salisbury, S. W. (2002): Crocodilians from the Lower Cretaceous (Berriasian) Purbeck Limestone Group of Dorset, southern England. Special Papers in Palaeontology, 68: 121-144.

Schellhorn, R., Schwarz-Wings, D., Maisch, M. W., Wings, O. (2009): Late Jurassic Sunosuchus (Crocodylomorpha, Neosuchia) from the Qigu Formation in the Junggar Basin (Xinjiang, China). Fossil Record, 12: 59-69.

Schudack, M. E. (2000): Geological setting and dating of the Guimarota-beds. In: Martin, T., Krebs, B. (eds.), Guimarota, a Jurassic ecosystem. Verlag Dr Friedrich Pfeil, München: 21-26.

Schwarz, D., Fechner, R. (2008): The first dentary of Lisboasaurus (Crocodylomorpha, ?Mesoeucrocodylia) from the Lower Cretaceous (Barremian) of Uña, Cuenca Province, Spain. Journal of Vertebrate Paleontology, 28: 264-268. http://dx.doi. org/10.1671/0272-4634(2008)28[264:TFDOLC]2.0.CO;2

Schwarz, D., Salisbury, S. (2005): A new species of Theriosuchus (Atoposauridae, Crocodylomorpha) from the Late Jurassic (Kimmeridgian) of Guimarota, Portugal, Geobios, 38: 79-802.

Scotese, C. R. (2000): Paleomap Project, Climate History. http:// www.scotese.com/climate.htm.

Seiffert, J. (1973): Upper Jurassic lizards from Central Portugal. Contribução para o Conhecimento da Fauna do Kimeridgiano da Mina de Lignito Guimarota (Leiria, Portugal). Memórias dos Serviços geológicos de Portugal (nov.ser.), 22: 7-85. 
Sha, J.G., Li, L., Chen, S.W., Matsukawa, M. (2006): Some Lower Cretaceous nonmarine bivalves from fluvio-lacustrine deposits bearing dinosaur fossils in Mongolia and northeast China. Cretaceous Research, 27: 262-278.

Sigogneau-Russell, D. (1981): Présence d'un nouveau Champsosauridé dans le Cretace supérieur de Chine. Comptes Rendus Académie des Sciences, Paris, 292: 1-4.

Sigogneau-Russell, D. (1985): Definition of the type-species of Simoedosaurus, S. lemoinei Gervais, 1877 (Choristodera, Reptilia). Journal of Paleontology, 59: 766-767.

Sigogneau-Russell, D., Efimov, M. (1984): Un Choristodera (Eosuchia?) insolite du Crétacé Inférieur de Mongolie. Palaontologische Zeitschrift, 58: 279-294.

Sigogneau-Russell, D., de Heinzelin, J. (1979): Les champsosaures europeens: mise au point sur le champsosauride d'Erquelinnes (Landenien inférieur, Belgique), Annales de Paleontologie (Vertebres), 65: 93-154.

Sigogneau-Russell, D., Russell, D. E. (1978): Etude osteologique du reptile Simoedosaurus (Choristodera). Annales de Paleontologie (Vertebres), 64: 1-84.

Simpson, G. G. (1930): Allognathosuchus mooki, a new crocodile from the Puerco Formation. American Museum Novitates, 445: 1-16.

Skutschas, P. P. (2008): A choristoderan reptile from the Lower Cretaceous of Transbaikalia. Neues Jahrbuch für Geologische und Palaontologische Abhandlungen, 247: 63-78. http://dx.doi. org/10.1127/0077-7749/2008/0247-0063

Storrs, G. W., Gower, D. J. (1993): The earliest possible choristodere (Diapsida) and gaps in the fossil record of semi-aquatic reptiles. Journal of the Geological Society, London, 150: 11031107.

Storrs, G. W., Gower, D. J., Large, N. F. (1996): The diapsid reptile Pachystropheus rhaeticus, a probable choristodere from the Rhaetian of Europe. Palaeontology, 39: 323-349.

Sweetman, S. C. (2009): The palaeoecology and preservation of a remarkable terrestrial vertebrate assemblage from the Barremian (Early Cretaceous) of Southern Britain. Mesozoic Terrestrial Ecosystems and Biota of Abstracts -10th Meeting, Teruel, Spain (September 17-19, 2009).

Tarduno, J. A., Brinkman, D. B., Renne, P. R., Cottrell, R. D., Scher, H., Castillo, P. (1998): Evidence for extreme climatic warmth from the late Cretaceous Arctic vertebrates. Science: 2241-2244. http://dx.doi.org/10.1126/science.282.5397.2241
Tsubamoto, T., Rougier, G. W., Isaji, S., Manabe, M., Forasiepi, A. M. (2004): New Early Cretaceous spalacotheriid "symmetrodont" mammal from Japan. Acta Palaeontologica Polonica: 49: 329-346.

Turner, C. E., Peterson, F. (2004): Reconstruction of the Upper Jurassic Morrison Formation extinct ecosystem - a synthesis. Sedimentary Geology, 167: 309-355.

Vandermark D., Tarduno J. A., Brinkman D. B. (2007): A fossil champsosaur population from the high Arctic: implications for Late Cretaceous paleotemperatures. Palaeogeography, $\mathrm{Pa}$ laeoclimatology, Palaeoecology, 248: 49-59. http://dx.doi. org/10.1016/j.palaeo.2006.11.008

Vakhrameev, V. A. (1978): The climates of the northern hemisphere in the Cretaceous in the light of paleobotanical data. $\mathrm{Pa}$ leontological Journal, (1978): 143-154.

Venczel, M., Gardner J. D. (2005): The geologically youngest albanerpetontid amphibian, from the lower Pliocene of Hungary. Palaeontology, 48: 1273-1300. http://dx.doi.org/10.1111/ j.1475-4983.2005.00512.x

Walker, A. D. (1970): A revision of the Jurassic reptile Hallopus victor (Marsh), with remarks on the classification of crocodiles. Philosophical Transactions of the Royal Society of London, 257: 323-372. http://dx.doi.org/10.1098/rstb.1970.0028

Wermuth, H. (1964): Das Verhaltnis zwischen Kopf-, Rumpf- und Schwanzlange bei den rezenten Krokodilen. Senckenbergiana Biologia, 45: 369-385.

Wu, X. -C. (2005): Crocodylians. In: P. J. Currie, E. B. Koppelhus (eds), Dinosaur Provincial Park, A Spectacular Ancient Ecosystem Revealed. Indiana University Press, Indianapolis: 277-291.

Wu, X. -C., Brinkman D. B, Lü, J. -C. (1994): A new species of Shantungosuchus from the Lower Cretaceous of Inner Mongolia, northern China, with comments on S. chuhsienensis Young, 1961 and the phylogenetic position of the genus. Journal of Vertebrate Paleontology, 14: 210-229. http://dx.doi.org/10.10 $\underline{80 / 02724634.1994 .10011553}$

Wu, X. -C., Sues, H. -D, Brinkman, D. B. (1996): An atoposaurid neosuchian (Archosauria: Crocodyliformes) from the Lower Cretaceous of Inner Mongolia (People's Republic of China). Canadian Journal of Earth Sciences, 33: 599-605.

Yabe, A., Terada, K., Sekido, S. (2003): The Tetori-type flora, revisited: a review. Memoir of the Fukui Prefectural Dinosaur Museum, 2: 23-42. 